\title{
Beyond Pairs:
}

\section{Definition and Interpretation of Third-Order Structure in Spatial Point Patterns}

\section{Chiho Kaito $^{1,2,3}$, Ulf Dieckmann ${ }^{1}$, Akira Sasaki²,1 and Fugo Takasu ${ }^{3}$}

${ }^{1}$ Evolution and Ecology Program, International Institute for Applied Systems Analysis (IIASA), 2361 Laxenburg, Austria

${ }^{2}$ Department of Evolutionary Study of Biosystems, SOKENDAI (The Graduate University for Advanced Studies), Shonan Village, Hayama, Kanagawa 240-0193, Japan

${ }^{3}$ Department of Information and Computer Sciences, Nara Women's University, KitaUoya Nishimachi, Nara 630-8506, Japan

Corresponding author: Fugo Takasu

E-mail: takasu@ics.nara-wu.ac.jp

Address: Department of Information and Computer Sciences, Nara Women's

University, Kita-Uoya Nishimachi, Nara 630-8506, Japan

Tel \& Fax: +81-742-20-3983 


\section{Abstract}

Spatial distributions of biological species are an important source of information for understanding local interactions at the scale of individuals. Technological advances have made it easier to measure these distributions as spatial point patterns, specifying the locations of individuals. Extensive attention has been devoted to analyzing the secondorder structure of such point patterns by focusing on pairs of individuals, and it is well known that the local crowdedness of individuals can thus be quantified. Statistical measures such as a point pattern's pair correlation function or Ripley's K function show whether a given point pattern is clumped (excess of short-distance pairs) or overdispersed (shortage of short-distance pairs). These notions are naturally defined in comparison with control patterns exhibiting complete spatial randomness, i.e., an absence of any spatial structure. However, there is no rational reason why the analysis of point patterns should stop at the second order. In this paper, we focus on triplets of individuals in an attempt to quantify and interpret the third-order structure of a point pattern. We demonstrate that point patterns with "bandedness", in which individuals are primarily distributed within bands, can be detected by an excess of thinner triplets at a characteristic spatial scale linked to the band's width. In this context, we show how the generation of control patterns as a reference for gauging a test pattern's triplet frequencies is critical for defining and interpreting the third-order structure of point patterns. Since perfect information on a point pattern's second-order structure typically suffices for its unique reconstruction (up to translation, rotation, and reflection), we conjecture that it is essential to minimally coarse-grain such second-order information before using it to generate control patterns for identifying a point pattern's third-order structure. We recommend the further exploration of this conjecture for future studies.

Keywords: Mapped point data, pair correlations, spatial configuration, spatial ecology, triplets 


\section{Introduction}

Biological species exhibit a variety of distributions over space. In spatial ecology, critical roles of space have been highlighted and it has been widely recognized that observing the spatial distributions of a species gives us a rich array of information about local interactions within and between species (Tilman and Kareiva 1997, Dieckmann et al. 2000). The spatial distribution, or spatial pattern, of a species can be measured using various census methods and it is often quantified as quadrant count data, transect data, or mapped point data, etc. Recently, mapped point data in which the locations of individuals of a species are explicitly specified is becoming more popular and easier to obtain as remote sensing and GIS/GPS technologies have been advanced and applied to spatial ecology (Roughgarden et al. 1991, Kerr and Ostrovsky 2003). Especially in plant and forest ecology, such mapped point data, or point patterns, have been accumulating. Extensive attention has been devoted to extract the biologically significant information hidden in a given point pattern, in order to understand the processes determining how individual plants are distributed over space through seed dispersal and local interactions (Watt 1947, Pielou 1969, Grieg-Smith 1983, Shimatani and Kubota 2004, Wiegand et al. 2007a, b, Law et al. 2009).

Any point pattern with $N$ points in two-dimensional space is uniquely represented as a "point" in $2 \mathrm{~N}$ dimensional space. Dealing directly with such a high dimensional space, however, becomes intractable as the number of points $N$ increases. Alternatively, approaches approximating the dynamics of spatial configuration densities represent a new departure in spatial ecology (Bolker and Pacala 1997, 1999, Dieckmann et al. 2000, Law et al. 2003): any point pattern can be characterized using spatial configuration densities that describe the densities of points, pairs, triplets, etc. As the number of points defining such configurations increases (points: 1; pairs: 2; triplets: 3; etc.), successive orders of information about a spatial point pattern are revealed (Dieckmann et al. 2000). 
The singlet density captures the most parsimonious information about a point pattern, describing its first-order structure by the density of points, i.e., by the number of points divided by the area over which they are distributed. A point pattern that only possesses first-order structure is generated by the homogeneous Poisson process with an intensity parameter given by the singlet density. In such a pattern, points occur completely randomly and independently from each other, realizing complete spatial randomness, or CSR (Cressie 1993). While the singlet density accounts for the abundance of points in a pattern, it obviously cannot capture any information about how these points are distributed in space. For capturing such information, a pattern's higher-order spatial structure has to be considered.

In particular, in order to capture information about the local crowdedness (clustering, aggregation) of points in a pattern, we need to focus on the pattern's second-order structure, defined by the densities of pair configurations. Specifically, a point pattern's pair density is defined as the spatial density of pairs of points in dependence on the vector describing their displacement (Dieckmann et al. 2000). In an sufficiently large isotropic point pattern, which exhibits no bias for any particular direction, the density of pairs is uniquely determined by the distance between them. For such patterns, measuring distances among pairs and establishing the distribution of those inter-point distances across all pairs therefore suffices to characterize a pattern's second-order structure. The pair density carries essentially the same information on a pattern's second-order structure as other basic summary statistics often used in point-pattern analyses, such as the pair correlation function, Ripley's K function, and O-ring statistics; all of these focus on the distribution of distances in pairs (Ripley 1988, Diggle 2003, Wiegand and Moloney 2004). By comparing the distribution of pair distances in a given point pattern to those expected under CSR with the same first-order structure (the same singlet density), we can discriminate whether or not the point pattern in focus significantly differs from CSR. More specifically, we can estimate the degree of clustering of points: a pattern is called 
clustered if it possesses an excess of pairs at short distances, whereas it is called regular, or overdispersed, when it exhibits a shortage of short-distance pairs (Turner 1989, Levin 1992, Dale 1999, Liebhold and Gurevitch 2002). By focusing on intraspecific (interspecific) pairs, in which the two individuals forming a pair belong to the same (different) species, salient features of intraspecific (interspecific) interactions at local spatial scale can be inferred (Dieckmann et al. 2000, Law and Dieckmann 2000).

In spatial ecology, measuring the first- and second-order structure (i.e., the singlet and pair densities) of point patterns has been a major interest. Approaches focusing on pairs have been established as powerful tools for quantifying key characteristics of observed point patterns and for unveiling local biological processes underlying such patterns (Diggle 2003, Law et al. 2009). In tropical forest studies, in particular, these approaches have been successfully applied, providing novel insights about local ecological interactions such as neighborhood competition between trees (Condit 1998). In response to the resultant increased needs for spatial point-pattern analyses, convenient statistical libraries such as "spatstat" (http://www.spatstat.org/spatstat/) are available in $\mathrm{R}$ (http://www.R-project.org/) to analyze the second-order structure of point patterns.

Approaches based on first- and second-order structure, however, may not characterize a point pattern completely. To describe higher-order structures that pair-based approaches may fail to capture, it is necessary to consider spatial configurations beyond pairs. As a first step in this direction, it is natural to focus on triplets, describing triangular spatial configurations comprising three points. We expect that the triplet density, defined as the density of triplets with a given triangular configuration, provides a useful measure to account for the next level of complexity in spatial structure. This will allow identifying and quantifying interesting features in the third-order structure of point patterns beyond what can be assessed based on pairs. 
Earlier studies have already explicitly called for extending the analysis of point pattern to triplet densities. Law et al. (2009) commented that "There is no intrinsic reason why the spatial analysis should not continue beyond second order to the density of triplets, quadruplets, etc. (Schladitz and Baddeley 2000). However, as yet there has been little exploration of higher-order spatial moments and the interpretation of these is not straightforward."

So far indeed, only a very few attempts have been made to utilize the triplet density for point-pattern analyses (Schladitz and Baddeley 2000, Bhanu and Tan 2001, Orlov et al. 2001). Dale and Powell (2001) describe new methods for the detection of patches and gaps in spatial point patterns based on counting points in the circumcircles of triangles defined by triplets. Although the triplet densities have been defined (Bolker and Pacala 1997, Law and Dieckmann 2000, Illian et al. 2008), no study yet has explicitly explored the significance of information captured by triplet configurations. Thus, how to analyze and interpret spatial configurations of triplets remain widely open questions.

The scarcity of published analyses focusing on triplets can perhaps be attributed to the greater complexity in quantifying the configurational characteristics of a triplet. While a pair is uniquely characterized by a single vector, characterizing a triplet requires two vectors. In two-dimensional space, this implies the need to specify two numbers (pairs) or four numbers (triplets). When a pattern is isotropic, these counts reduce to one (pairs) and three (triplets), as the one degree of freedom describing the pattern's absolute rotational position is stripped away.

In this paper, we respond to the challenge to quantify the third-order structure of point patterns and explore the significance of the extra information such structure conveys. In this way, we aim to introduce triplet densities as a novel target of study in spatial ecology. For this purpose, we explore the triplets densities of a variety of point patterns that possess 
clearly distinguishable higher-order structure in the form of "bandedness", in which points are distributed primarily within bands. In the Methods, we describe our main idea and introduce a way to define and interpret triplet densities. In the Results, we then apply our idea to several classes of point patterns and examine the resultant characteristics of triplet configurations. In the Discussion, we examine the prospects of using triplet densities as a new tool for capturing the next level of complexity in point patterns.

\section{Methods}

\subsection{Overview}

Suppose that, after the singlet density and pair density of a point pattern have been analyzed, triplet densities are assessed from the point pattern to reveal the extra information contained in the pattern's third-order structure. What additional insights can this yield?

Our main idea for interpreting triplet densities is straightforward. To explain it, we consider a point pattern that has points clustered in bands of a characteristic width. When assessing the densities of the triangles formed by all possible triplets of a particular range of spatial scales, comparable to the width of these bands, there will be disproportionally more thin triangles and less near-equilateral ones than expected in point patterns without such bandedness. An illustration is shown in Figure 1:

- In this point pattern, triplets of any shape are found at large scale (Figure 1a).

- Thinner triplets predominate at the intermediate scale that is comparable to the width of the shown band (Figure 1b).

- At smaller scale yet, triplets of any shape are again found (Figure 1c).

An excess of thinner triplets at a certain spatial scale will thus indicate the presence of bandedness in a point pattern, and the scale at which thinner triplets start to predominate will carry information about the width of the band in the point pattern. 
To make this idea applicable, the following three open questions have to be addressed:

- First, we need to specify how to measure a triplet's scale and shape.

- Second, using the chosen measures of scale and shape in determining a given test pattern's triplet density, we need to establish a criterion for assessing the excess of thinner triplets, i.e., we need to define control patterns to be used as a reference against which to gauge an excess of thinner triplets in the test pattern.

- Third, we need to specify how to quantify the excess of thinner triplets and how to infer from that the characteristic triplet scale at which this excess occurs.

In the next sections, we describe our approach to each of these questions step by step.

Figure 1.

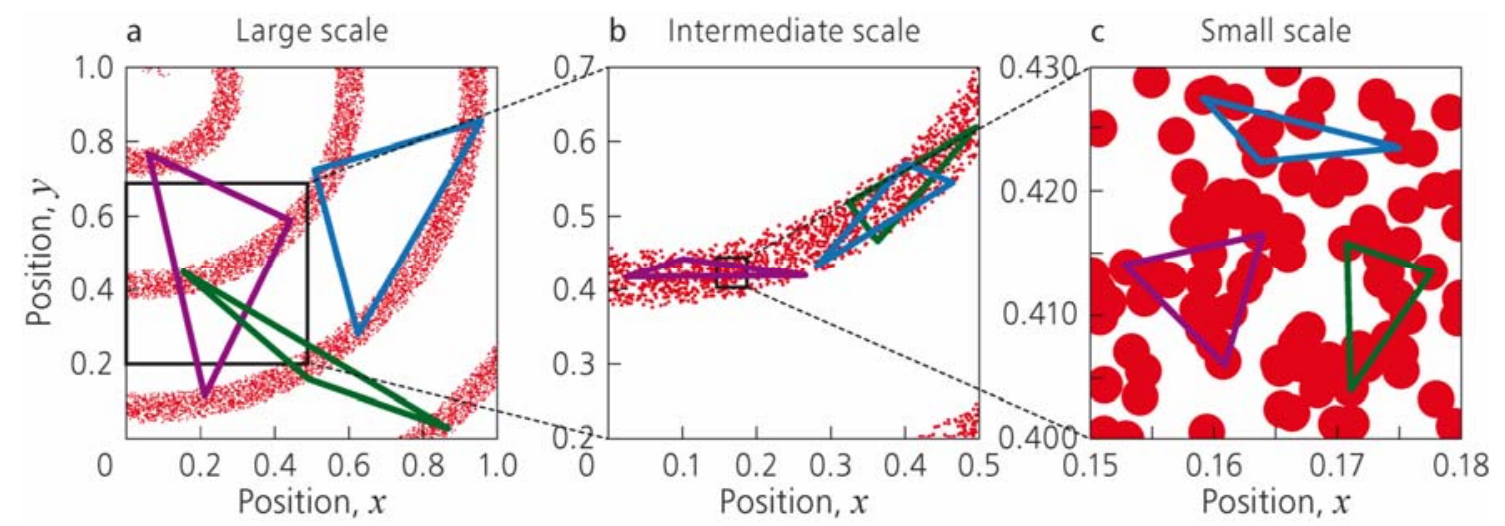

\subsection{Measures of triplet scale and shape}

Let us consider a triplet of three distinct points $i, j, k$. As shown in Figure 2, these points define three edges $L_{i}, L_{j}, L_{k}$ and three angles $\theta_{i}, \theta_{j}, \theta_{k}$. On this basis, we now introduce measures that quantify the triplet's scale and shape:

- As a scale measure $S$ that describes how large the triplet is, we use the arithmetic mean of two edges $\left(L_{j}+L k\right) / 2$. When magnifying any triangle by a given factor, this scale measure is increased by the same factor.

- As a shape measure $I$ that describes how thin a triplet is: for this purpose, we use the angle $\theta_{i}$ surrounded by the two edges $L_{j}$ and $L_{k}$. When magnifying any triangle by a given factor, this shape measure is left invariant. 
Any triplet thus has three matched measures of scale and shape: $\left(\left(L_{j}+L_{k}\right) / 2, \theta_{i}\right),\left(\left(L_{k}+\right.\right.$ $\left.\left.L_{i}\right) / 2, \theta_{j}\right)$, and $\left(\left(L_{i}+L_{j}\right) / 2, \theta_{k}\right)$. In all of these, the two edges used in the scale measure surround the angle used as the shape measure. The shape measure I can take values within the range $0 \leq I \leq \pi$. Equality holds when the triangle is degenerate (i.e., when the three points lie on a straight line). The thinner a triangle becomes, the more closely its three shape measures $I$ approach 0 (for two of them) or $\pi$ (for one of them).

For the sake of tractability, it is often advisable to discretize the aforementioned measures of scale and shape. Thus, a given value of the scale measure $S$ falls into the sth bin of the scale measure, and a given value of the shape measure $I$ falls into the $i$ th bin of the shape measure.

Figure 2.

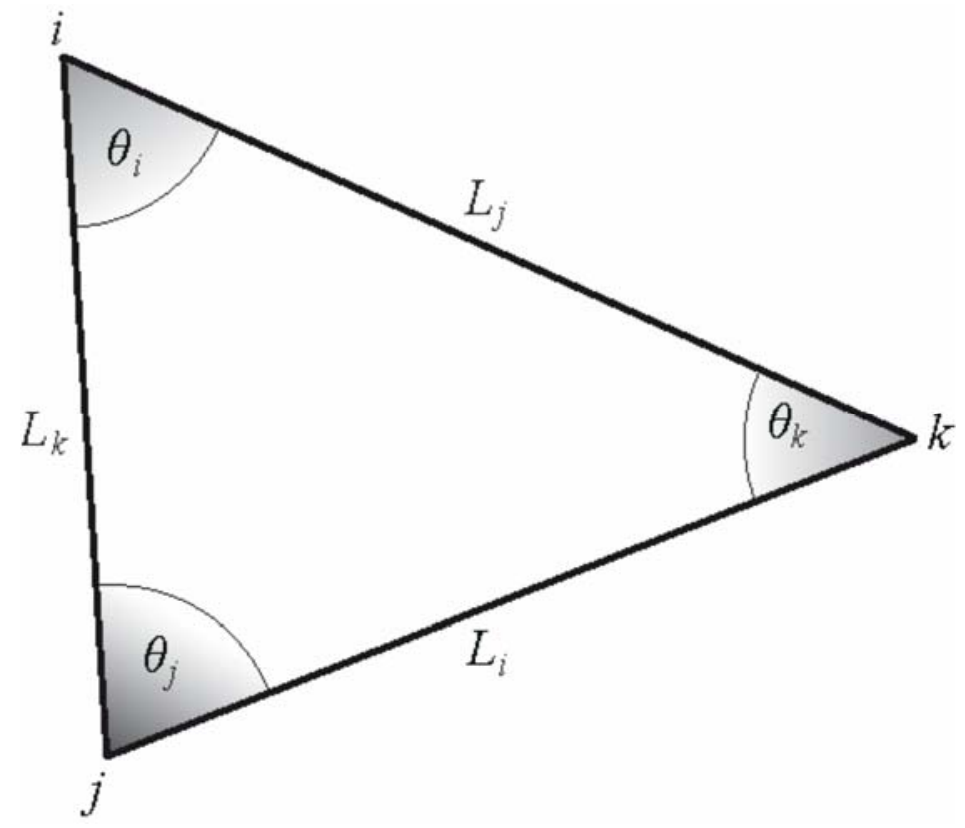

To start determining a given test pattern's third-order structure, we sample a large number of its triplets. For each triplet, we translate the matched measures $\left(\left(L_{j}+L_{k}\right) / 2, \theta_{i}\right),\left(\left(L_{k}+\right.\right.$ $\left.\left.L_{i}\right) / 2, \theta_{j}\right)$, and $\left(\left(L_{i}+L_{j}\right) / 2, \theta_{k}\right)$ into the corresponding bins $\left(s_{1}, i_{1}\right),\left(s_{2}, i_{2}\right)$, and $\left(s_{3}, i_{3}\right)$. In this way, we obtain the triplet-density matrix $\mathbf{T}=\left(T_{s i}\right)$, where $T_{s i}$ is the number of triplets that have a scale and a shape lying within the sth and ith bin of the scale and the shape 
measure, respectively, where both $s$ and $i$ run from 1 to the respective numbers of bins. In practice, $\mathbf{T}=\left(T_{s i}\right)$ is computed by starting with a matrix of $0 \mathrm{~s}$ and incrementing $T_{s_{1} i_{1}}$, $T_{s_{2} i_{2}}$, and $T_{s_{3} i_{3}}$ by 1 for each sampled triplet.

As the number of triplets systematically increases with their scale, we normalize the triplet-density matrix $\mathbf{T}$ to obtain the matrix $\mathbf{T}^{*}=\left(T_{s i}^{*}\right)$ that in each row $s$ contains the frequency distribution of shape measures at that scale (consequently, for each scale $s$ the row elements $T_{s i}^{*}$ sum to 1 over all is). The triplet-frequency matrix $\mathbf{T}^{*}$ thus captures the spatial configuration of triplets in the test pattern.

\subsection{Control patterns for defining third-order structure}

We next need to specify how to gauge an excess of thinner triplets in the test pattern. In pair-based approaches, the predominance of short-distance pairs in a test pattern is gauged against a CSR point pattern that has the same first-order structure (i.e., the same singlet density) as the test pattern. In our triplet-based approach, therefore, a control pattern against which the excess of thinner triplets is gauged should have the same first- and second-order structure as the test pattern.

In order to generate such a control pattern, we adopt the Metropolis-Hasting algorithm (Hastings 1970), hereafter MH, a Markov Chain Monte Carlo method. Starting from a CSR point pattern with the same singlet density as the test pattern, points are randomly shifted in space so that the distribution of inter-point distances converges to that of the test pattern within a given accuracy (which trades off with the required computational time; see Appendix A for details).

Once a control pattern is generated for a test pattern, the triplet-density matrix $\mathbf{T}^{\mathrm{c}}=\left(T^{\mathrm{c}}{ }_{s i}\right)$ and the triplet-frequency matrix $\mathbf{T}^{\mathrm{c}^{*}}=\left(T^{\mathrm{c}^{*}}{ }_{s i}\right)$ of the control pattern are obtained just as for the test pattern. 
On this basis, we define the test pattern's third-order structure by the difference in the triplet-frequency matrices of the test pattern and the control pattern, $\mathbf{T}^{*}$ and $\mathbf{T}^{\mathrm{c}^{*}}$. This yields the excess triplet-frequency matrix $\mathbf{T}^{\mathrm{e}}=\mathbf{T}^{*}-\mathbf{T}^{\mathrm{c}^{*}}$. If a test pattern has more triplets at scale $s$ and shape $i$ than expected based on its first- and second-order structure, $T_{s i}{ }_{s i}$

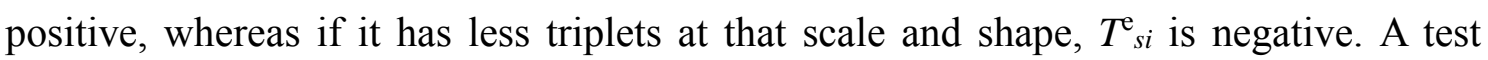
pattern's third-order structure can thus conveniently be visualized by showing its excess triplet-frequency matrix in the two dimensional space spanned by scale and shape.

\subsection{Quantification of the excess of thin triplets and definition of the characteristic triplet scale}

For a banded point pattern as shown in Figure 1, no third-order structure exists at scales much below the width of the considered band. Hence, the rows of the excess tripletfrequency matrix at those scales must contain values close to 0 . At scales comparable to the band's width, thinner triplets start to predominate in the test pattern when compared with the control pattern, so the corresponding rows of the excess triplet-frequency matrix must show positive values around 0 and $\pi$, and negative values in between. In order to define the characteristic triplet scale above which this systematic departure starts to emerge, we need to define a measure of the deviation of the test pattern from the control pattern in terms of the frequency distributions of shapes as a function of scale.

We use the Jensen-Shannon divergence to measure the divergence $D(S)=D_{\mathrm{JS}}\left(\mathbf{T}^{*}{ }_{s} \| \mathbf{T}^{\mathrm{c}^{*}}{ }_{s}\right)$ between the two frequency distributions $\mathbf{T}^{*}{ }_{s}$ and $\mathbf{T}^{\mathrm{c}^{*}}{ }_{s}$ of shapes as a function of scale $S$ (see Appendix B for details). Plotting $D(S)$ against $S$ enables us to quantify the characteristic scale at which the third-order structure specific to the test pattern starts to emerge.

To assess at which divergence threshold the characteristic triplet scale should be defined, 
we consider a range of test patterns with bands of different widths, for each of them calculate the squared difference between the actual width of the band and the estimated characteristic triplet scale defined by an assumed divergence threshold, and then obtain the optimal divergence threshold that minimizes the sum of squared differences.

In the following analysis, we explore a series of test patterns within the finite spatial range $0 \leq x \leq 1,0 \leq y \leq 1$. Specifically, we consider straight bands, arcs or curved bands, rings, spirals, and crosses of straight bands. The number of points (which here equals the singlet density, as the considered spatial range has an area of 1) is at least 1,000 in all of these test patterns. Sampling all triplets is computationally neither easy nor necessary, as the number of possible triplets increases in cubic order with the number of points. We always sample 10,000,000 triplets, which ensures that sampling variances in the triplet-frequency matrices $\mathbf{T}^{*}$ and $\mathbf{T}^{\mathrm{c}^{*}}$ are sufficiently small (except at the smallest scales) when using 100 bins for the scale measure $S(0 \leq S \leq 1)$ and 50 bins for the shape measure $I(0 \leq I \leq \pi)$.

\section{Results}

We first apply our approach to point patterns featuring straight bands of different widths, before comparing the results with those for qualitatively different classes of test patterns.

\subsection{Triplet frequencies of a rectangular band}

As a first example, we analyze a test pattern in which points form a rectangular band. Specifically, 3,000 points are distributed under CSR within a rectangular region of width $0.15(0.425 \leq x \leq 0.575$ and $0 \leq y \leq 1)$ (Figure 3a). A control pattern generated using the $\mathrm{MH}$ algorithm is shown in Figure 3b: by construction, this has the same first- and secondorder structure as the test pattern, which is confirmed by the comparison of pair densities shown in Figure 3c. 
The triplet frequencies of the test pattern and that of the control patterns averaged over 10 control patterns are shown in Figures $3 \mathrm{~d}$ and $3 \mathrm{e}$, respectively. The excess tripletfrequency matrix $\mathbf{T}^{\mathrm{e}}$ is shown in Figure $3 \mathrm{f}$. At small scales, the triplet frequencies in the test pattern are similar to those in the control patterns. But they start to deviate as the scale is increased, and at an intermediate scale, the excess of thinner triplets (with angles close to either 0 or $\pi$ ) becomes evident as a clear bimodality in the frequency distribution of angles. As the scale is further increased, this bimodality disappears again, as triplet frequencies start to become dominated by the boundary effects that inevitably occur for any point patterns limited to a finite spatial range. Therefore, we hereafter focus on scales $s$ smaller than about 0.5 .

The divergence $D(S)$, measuring the difference in the frequency distributions of triplet angles shown in Figures $3 \mathrm{~d}$ and $3 \mathrm{e}$ as a function of scale $S$ (Figure $3 \mathrm{~g}$ ), continuously grows as the scale is increased. For very small scales, below about $s=0.02$, this divergence also increases. This is an artifact caused by the finite number of points in the test pattern: because of the scarcity of very small-sized triplets, both in the test pattern and in the control patterns, the triplet frequencies at such small scales are noisy. This noise diminishes - and the resultant divergence eventually vanishes - with more points in the test pattern, a bigger spatial range of the test pattern, larger bins for the scale measure, and larger bins for the shape measure. 
Figure 3.
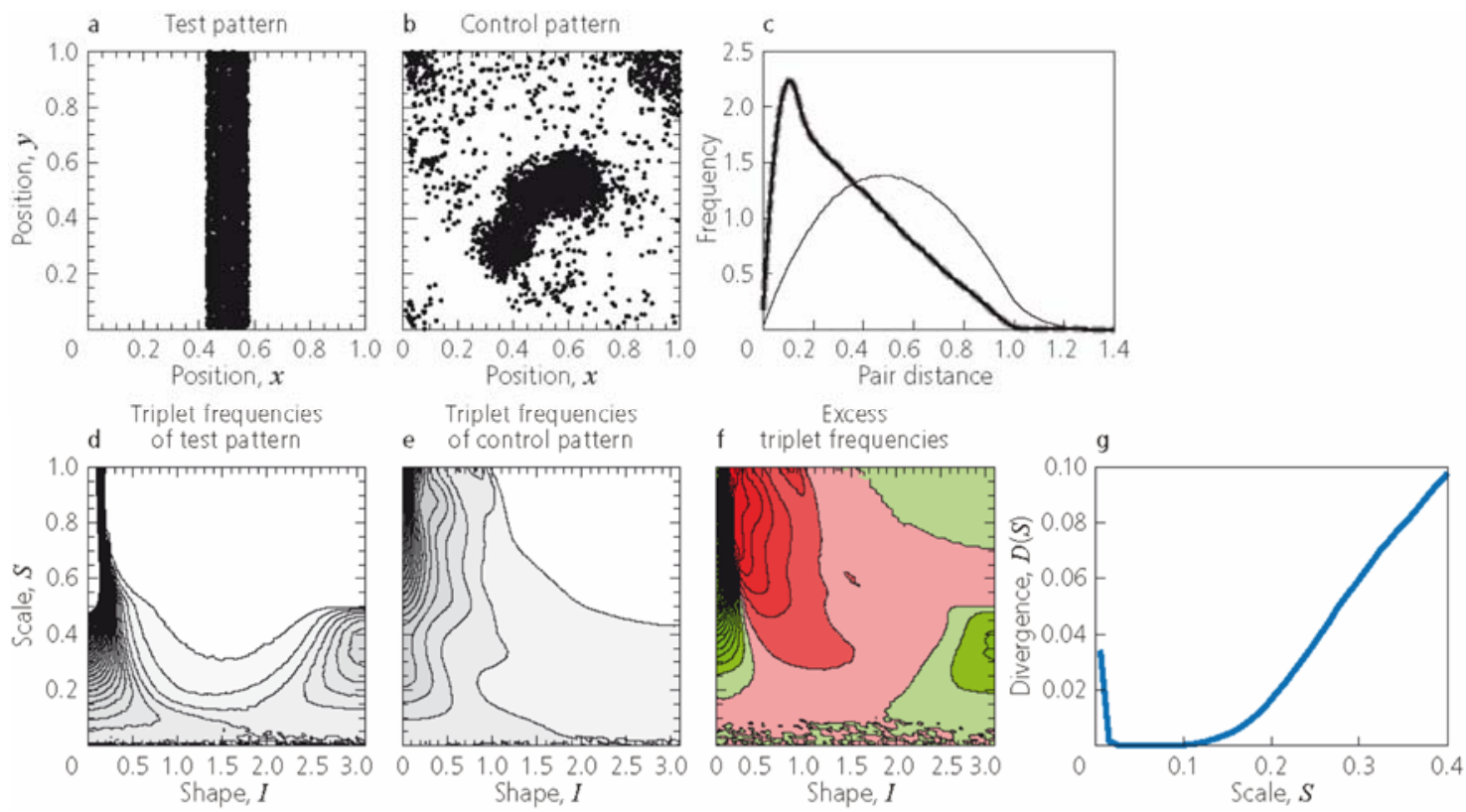

\subsection{Characteristic triplet scales of rectangular bands}

We next explore how the width of a rectangular band in a test pattern is reflected in the triplet-frequency matrix. For this purpose, we use five test patterns, each having a band of points distributed under CSR in rectangular regions of different width $(0.05,0.10,0.15$, 0.20, and 0.25), with the singlet density kept constant within the band (Figures 4a-e). For each of these test patterns, we generate ten control patterns, one of which is shown in Figures 4f-j. The resultant excess triplet frequencies are shown in Figures 4k-o.

If these bands of points had infinite vertical lengths $(-\infty<y<\infty)$ and singlet densities, the triplet frequencies at any given scale would be invariant under multiplying the band width and the scale by the same factor. For example, the triplet frequencies of a test pattern with a band of width $w^{\prime}=0.05(0.475<x<0.525,-\infty<y<\infty)$ at the scale $S^{\prime}$ would exactly match those of a test pattern with a band of width $w=3 \times 0.05=0.15$ $(0.425<x<0.575,-\infty<y<\infty)$ at the scale $S=3 S^{\prime}$. To see how this scaling applies despite the finite vertical lengths and singlet densities of the rectangular bands, we plot the divergence measure $D\left(S^{\prime}\right)$ of the test pattern with band width $w^{\prime}$ as a function of $S^{\prime}=S$ $w^{\prime} / w$, i.e., of the scale that has been rescaled relative to the band width of $w=0.15$ as a 
reference (Figure $4 u)$.

When rescaled in this manner, the divergence measures of the five test patterns with various band widths generally match each other well (Figure $4 \mathrm{u}$ ). They all show an initial decline to zero as the scale is increased from zero, reflecting the noise caused by the scarcity of small-sized triplets. They then start to rise as the scale is increased and the triplet frequencies in the test pattern and the control pattern start to deviate at the scale of the considered band width. Indeed, the results in Figure $4 \mathrm{u}$ suggest that we can approximately read off the widths of the analyzed bands (vertical arrow in Figure $4 u$ ) by considering when the deviance between test pattern and control pattern exceeds a certain threshold (horizontal line in Figure $4 \mathrm{u}$ ). For any test pattern, a characteristic triplet scale can thus be defined by the passing of such a divergence threshold. We now examine the optimal choice of this threshold for rectangular bands.

For a continuous range of candidate divergence thresholds, Figure $4 \mathrm{v}$ shows the resultant sum of squared differences between the actual reference band widths (always 0.15 after rescaling) and the estimated band widths, obtained with the following algorithm. (1) For all five considered band widths $w^{\prime}$, plot the divergence $D\left(S^{\prime}\right)$ against the scale $S^{\prime}=S w^{\prime} / w$ (Figure $4 \mathrm{u}$ ). (2) For all $w^{\prime}$ and all candidate values $D^{*}$ of the divergence threshold, calculate the scale $S^{\prime *}$ at which $D\left(S^{\prime *}\right)=D^{*}$. (3) For all $w^{\prime}$ and all $D^{*}$, calculate the squared difference between $S^{\prime *}$ and the reference band width 0.15 and sum these squared differences for all five band widths as a function of $D^{*}$ (Figure $4 \mathrm{v}$ ). We then estimate the optimal divergence threshold as the one that minimizes the sum of squares, which yields $D^{*}=0.0043($ Figure $4 \mathrm{v})$.

Using this optimal divergence threshold (horizontal lines in Figures 4p-t), we consider the divergence $D(S)$ in the original scale (curves in Figures $4 \mathrm{p}-\mathrm{t}$ ) to derive the estimated band widths (vertical lines in Figures $4 p-t)$. The results are plotted against the actual band 
widths in Figure 4w. We can thus see that all combinations of estimated and actual band widths nearly fall on the line with slope 1 . This demonstrates that the width of the rectangular bands can be precisely captured by the scale beyond which the distributions of triplet frequencies starts to deviate between the test and control patterns. 
Figure 4.
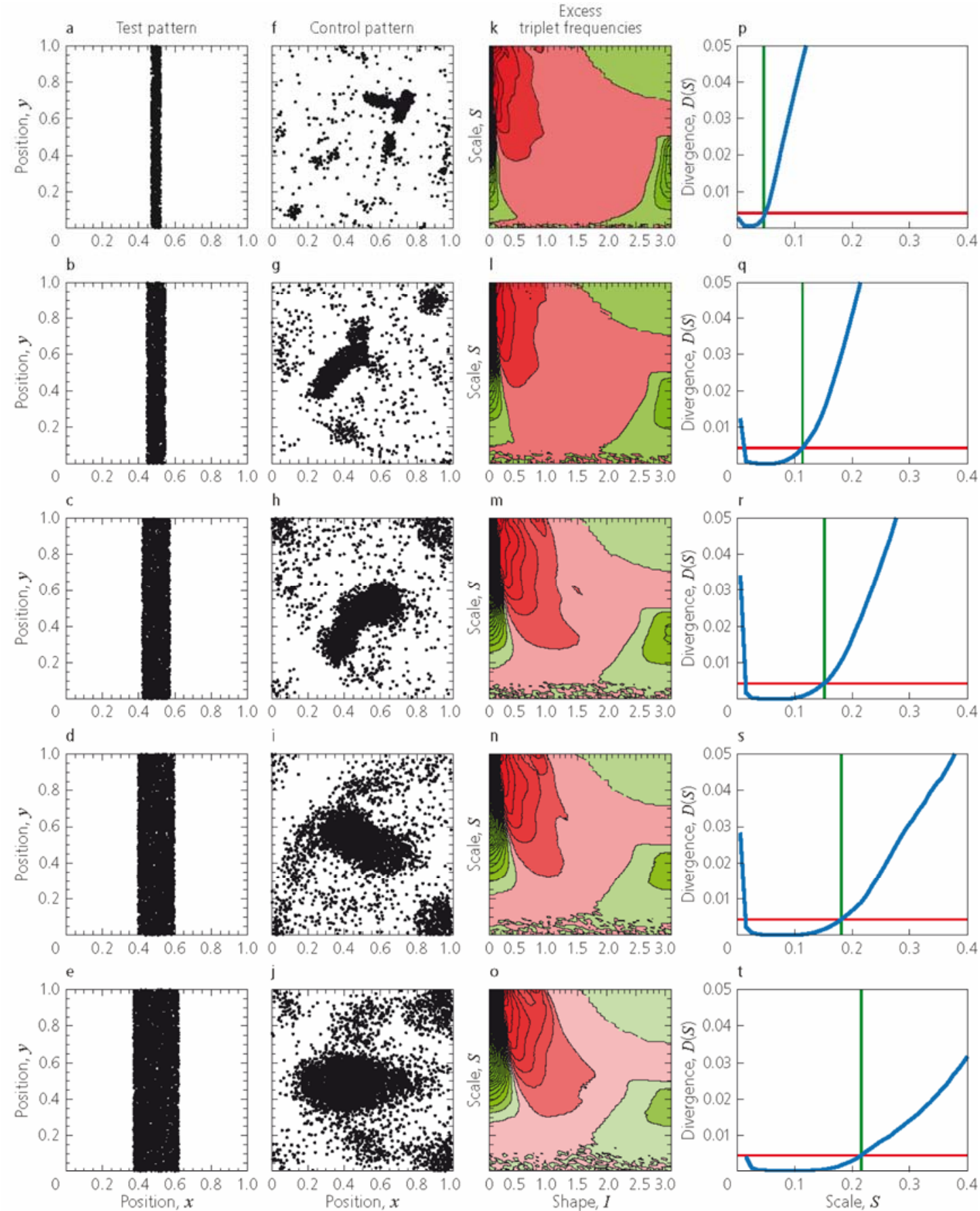

0
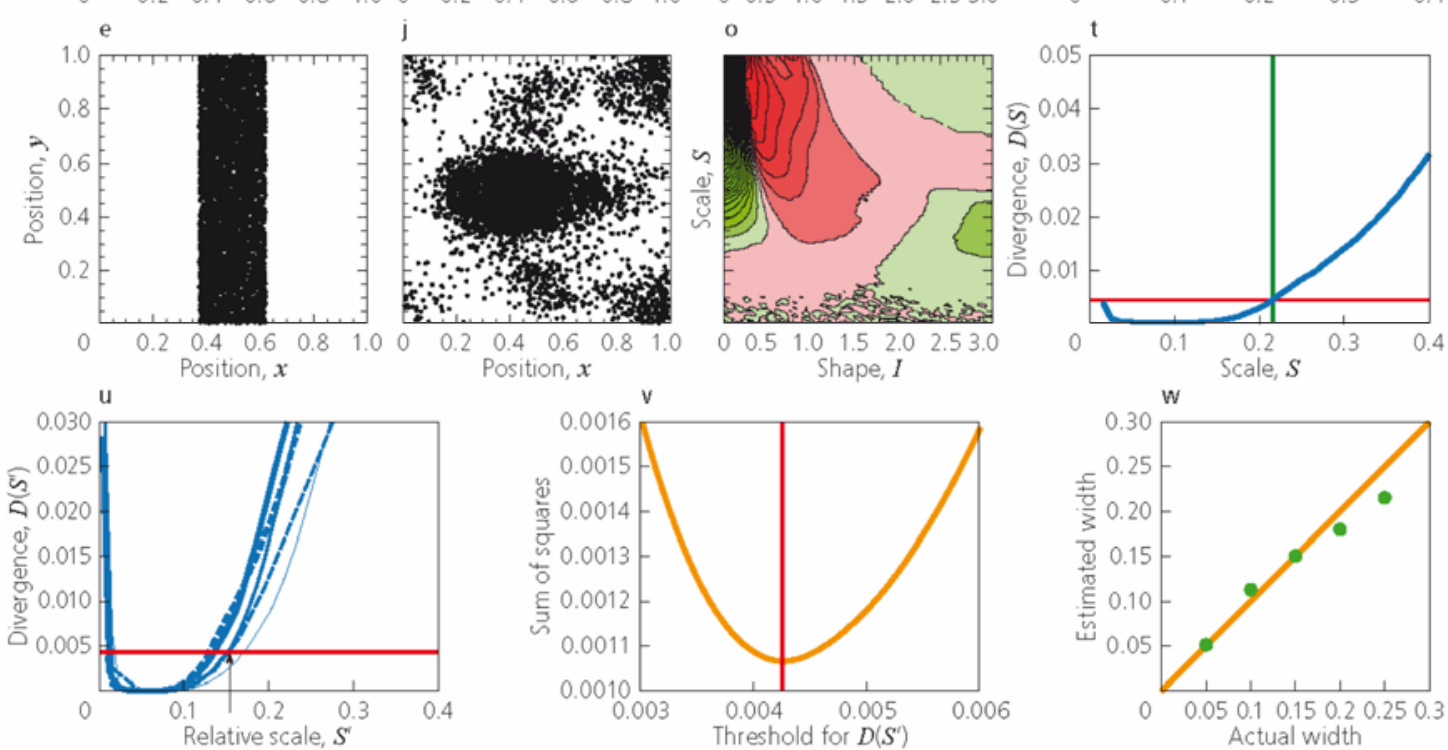


\subsection{Robustness of our approach applied to other test patterns}

In order to examine the robustness of our approach, we apply it to various other classes of test patterns that have clear bandedness with various band widths and band shapes. For each class of test pattern, we derive the optimal divergence threshold and use it to determine each test pattern's characteristic triplet scale. As a further robustness check, we also apply our approach to test patterns without bandedness.

\subsubsection{Arcs}

We next examine test patterns with arc-shaped bands of different widths (Figure 5). Figures 5a-e show five test patterns with widths of $0.05,0.10,0.15,0.20$, and 0.25 with the singlet density kept constant within each arc. Figures $5 \mathrm{f}-\mathrm{j}$ show corresponding control patterns. Figures 5k-o show the resultant excess triplet-frequency matrices. Bimodality in the frequency distribution of triplet angles, with peaks at 0 and $\pi$, occurs at intermediate scales. Figures $5 \mathrm{p}$-t show the divergence measure $D(S)$ and the optimal divergence threshold. Figure $5 \mathrm{u}$ shows the divergence measures $D\left(S^{\prime}\right)$ for all five test patterns as functions of the relative scale $S^{\prime}$ that is standardized to the reference width of 0.15 . Figure $5 \mathrm{v}$ shows the resultant optimal divergence threshold of 0.0046 that minimizes the sum of squared differences. Figure $5 \mathrm{w}$ shows the comparison of estimated widths and the actual widths. As we see, our approach again successfully estimates the widths of the bands forming the arcs: the estimated widths are nearly linearly related to the actual widths as long as the arcs are not too wide. 
Figure 5.
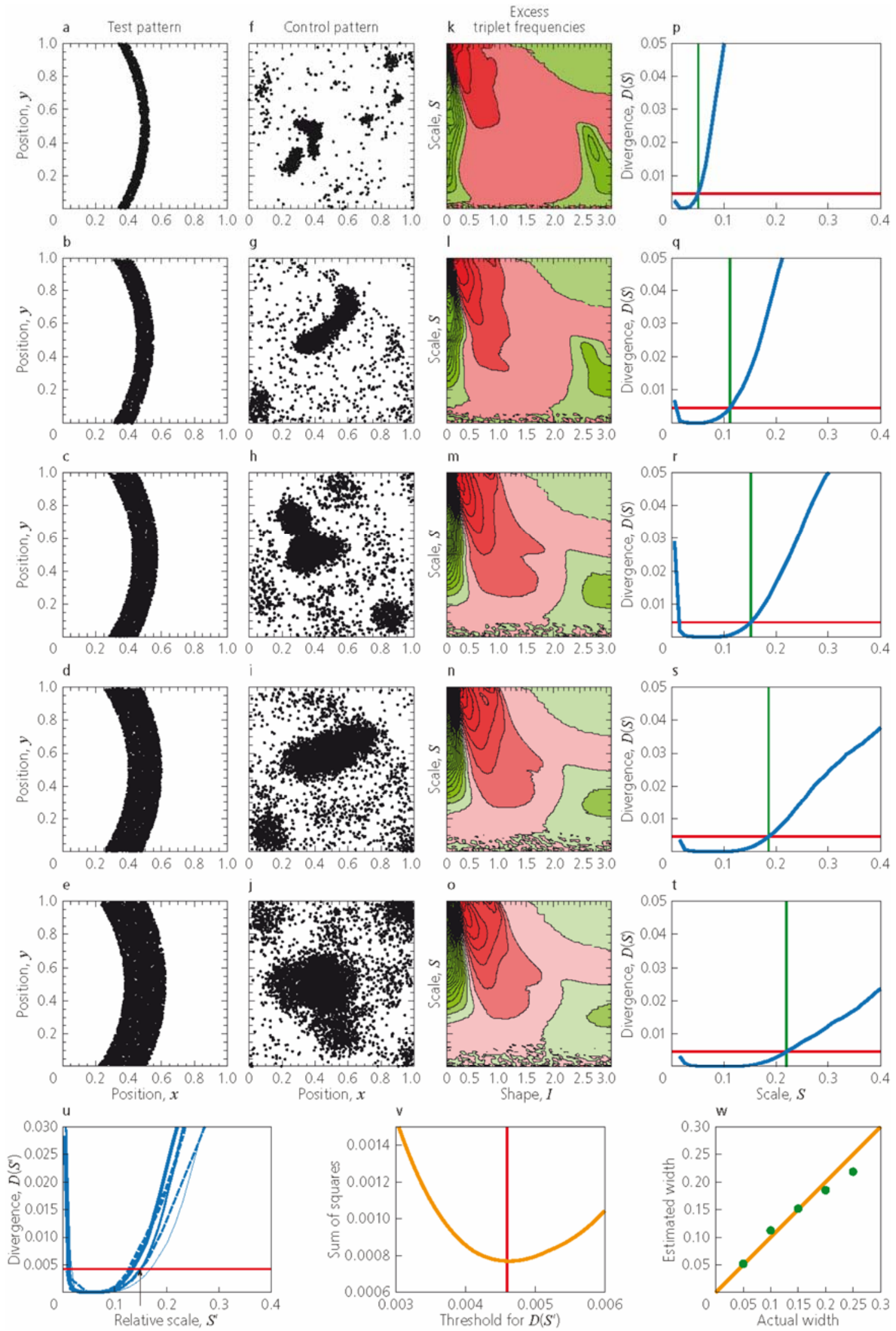


\subsubsection{Rings}

We next examine test patterns with ring-shaped bands of different widths (Figure 6). Figures 6a-e show five test patterns with widths of $0.05,0.07,0.09,0.10$, and 0.15 . Control patterns, excess triplet-frequency matrices, and the divergence measure $D(S)$ are shown in Figures 6f-j, 6k-o, and 6p-t, respectively. Figure 6 u shows $D\left(S^{\prime}\right)$ as functions of the relative scale $S^{\prime}$. Bimodality in the frequency distribution of triplet angles, with peaks at 0 and $\pi$, occurs at intermediate scales. The optimal divergence threshold is 0.0001645 according to Figure 6v, and estimated and actual widths are compared in Figure 6w. As we see, our approach successfully again estimates the widths of the bands forming the rings: the estimated widths are nearly linearly related to the actual widths, except for the narrowest width 0.05 . For the band width $0.05, D\left(S^{\prime}\right)$ is larger, across all short and intermediate scales $S^{\prime}$, than for the other band widths (Figure 6u), and we excluded this test pattern when deriving the optimal divergence threshold. This seemingly has been caused by the choice of the reference band width of 0.15 when deriving the optimal divergence threshold. Visual inspection suggests that a reference width of about 0.18 , not 0.15 , may work better. The curvature of the ring-shaped bands in the test pattern may be related to this result (see Discussion). Note also that the generated control patterns exhibit a ring-shaped pattern similar to the original test pattern, so the triplet frequencies in the control patterns are therefore similar to those in the test patterns. This is a likely reason for the very low optimal divergence threshold, as compared with those for rectangular bands and arc-shaped bands analyzed above (see Discussion). 
Figure 6.
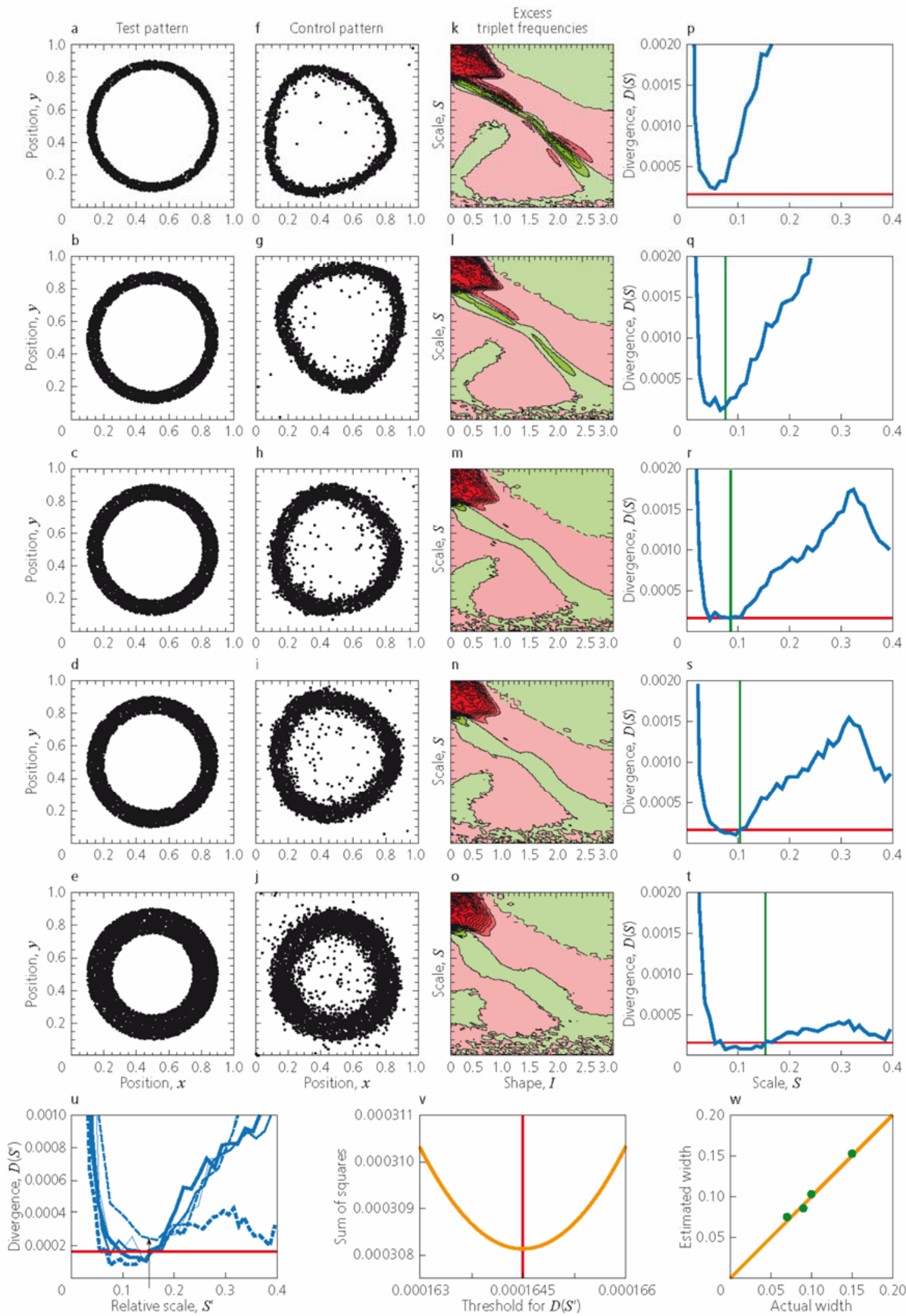


\subsubsection{Spirals}

We next examine test patterns with spiral-shaped bands of different widths (Figure 7). Figures $7 \mathrm{a}$-e show five test patterns with widths of $0.02,0.03,0.04,0.05$, and 0.06 . Control patterns, excess triplet-frequency matrices, and the divergence measure $D(S)$ are shown in Figures $7 \mathrm{f}-\mathrm{j}, 7 \mathrm{k}-\mathrm{o}$, and $7 \mathrm{p}-\mathrm{t}$, respectively. Figure $7 \mathrm{u}$ shows $D\left(S^{\prime}\right)$ as functions of the relative scale $S^{\prime}$. Bimodality in the frequency distribution of triplet angles, with peaks at 0 and $\pi$, occurs at intermediate scales for narrow widths. The optimal divergence threshold is 0.0122 according to Figure $7 \mathrm{v}$, and estimated and actual widths are compared in Figure 7w. As we see, our approach again successfully estimates the widths of the bands forming the spirals: the estimated widths are nearly linearly related to the actual widths. For the spiral with the broadest band, with a width of $0.06, D\left(S^{\prime}\right)$ is smaller, across all intermediate and large scales $S^{\prime}$, than for the other band widths (Figure $7 \mathrm{u}$ ), and we excluded this test pattern when deriving the optimal divergence threshold. 
Figure 7.
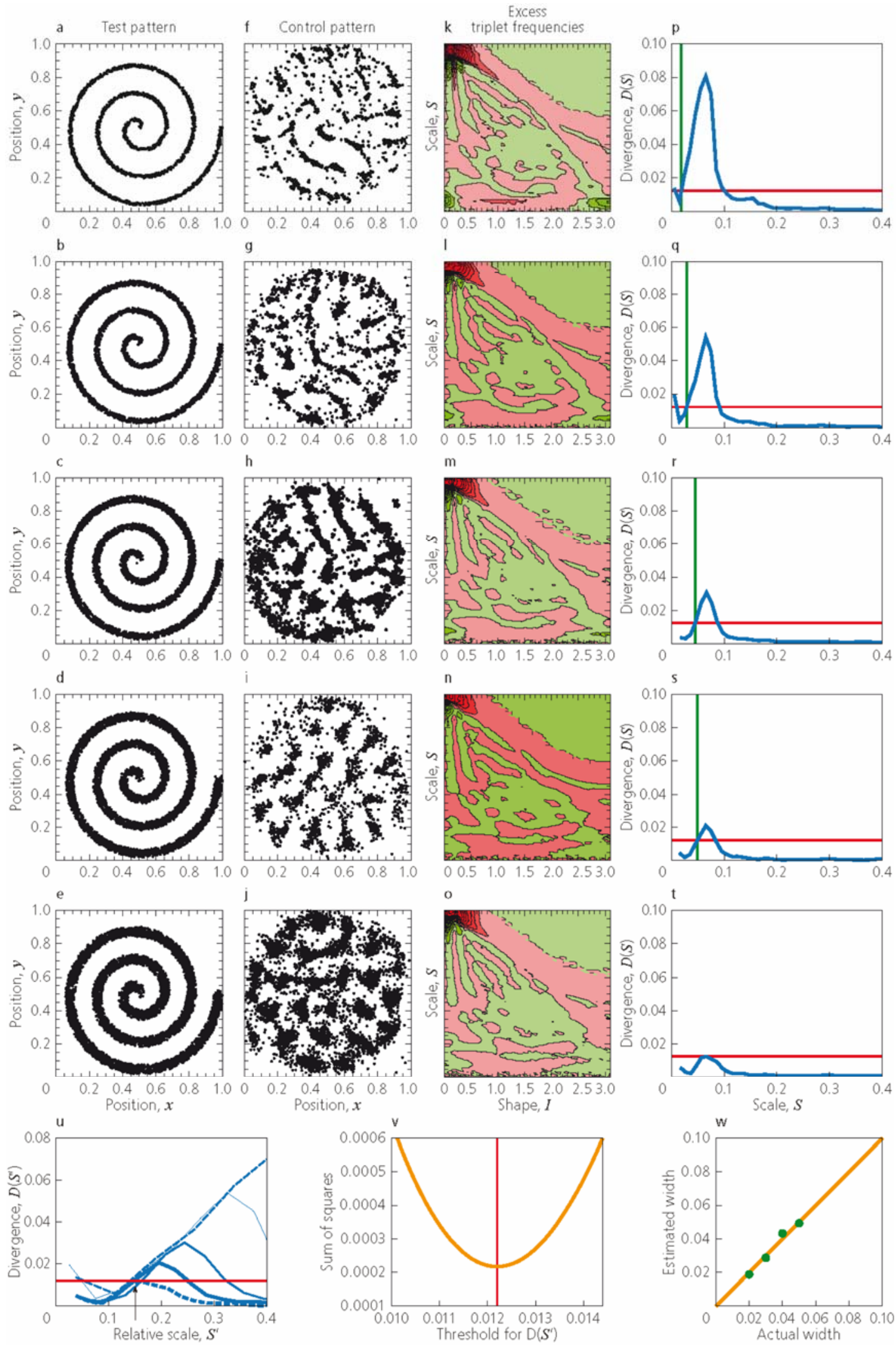


\subsubsection{Crosses}

We next examine test patterns with combinations of three rectangular bands (Figure 8). Figures 8a-e show five test patterns with the widths of $0.05,0.07,0.09,0.10$, and 0.15 . Control patterns, excess triplet-frequency matrices, and the divergence measure $D(S)$ are shown in Figures 8f-j, 8k-o, and 8p-t, respectively. Figure $8 \mathrm{u}$ shows $D\left(S^{\prime}\right)$ as functions of the relative scale $S^{\prime}$. Bimodality in the frequency distribution of triplet angles, with peaks at 0 and $\pi$, occurs at intermediate scales for narrow widths. The optimal divergence threshold is 0.00302 according to Figure $8 \mathrm{v}$, and estimated and actual widths are compared in Figure 8w. As we see, our approach again successfully estimates the widths in the combinations of three rectangular bands: the estimated widths are nearly linearly related to the actual widths, except for the broadest width 0.15 , which consequently was excluded when deriving the optimal divergence threshold. 
Figure 8.
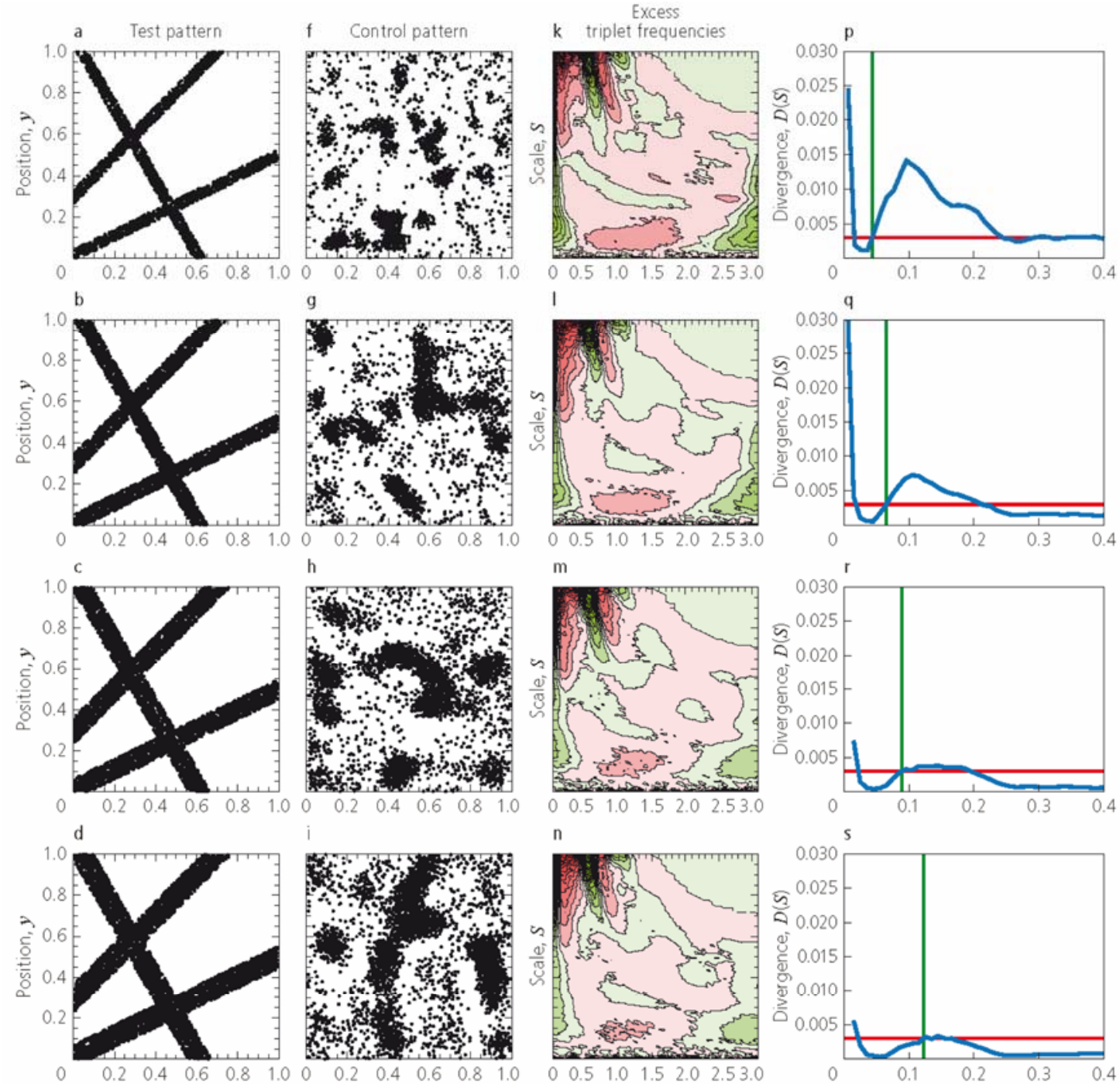

n
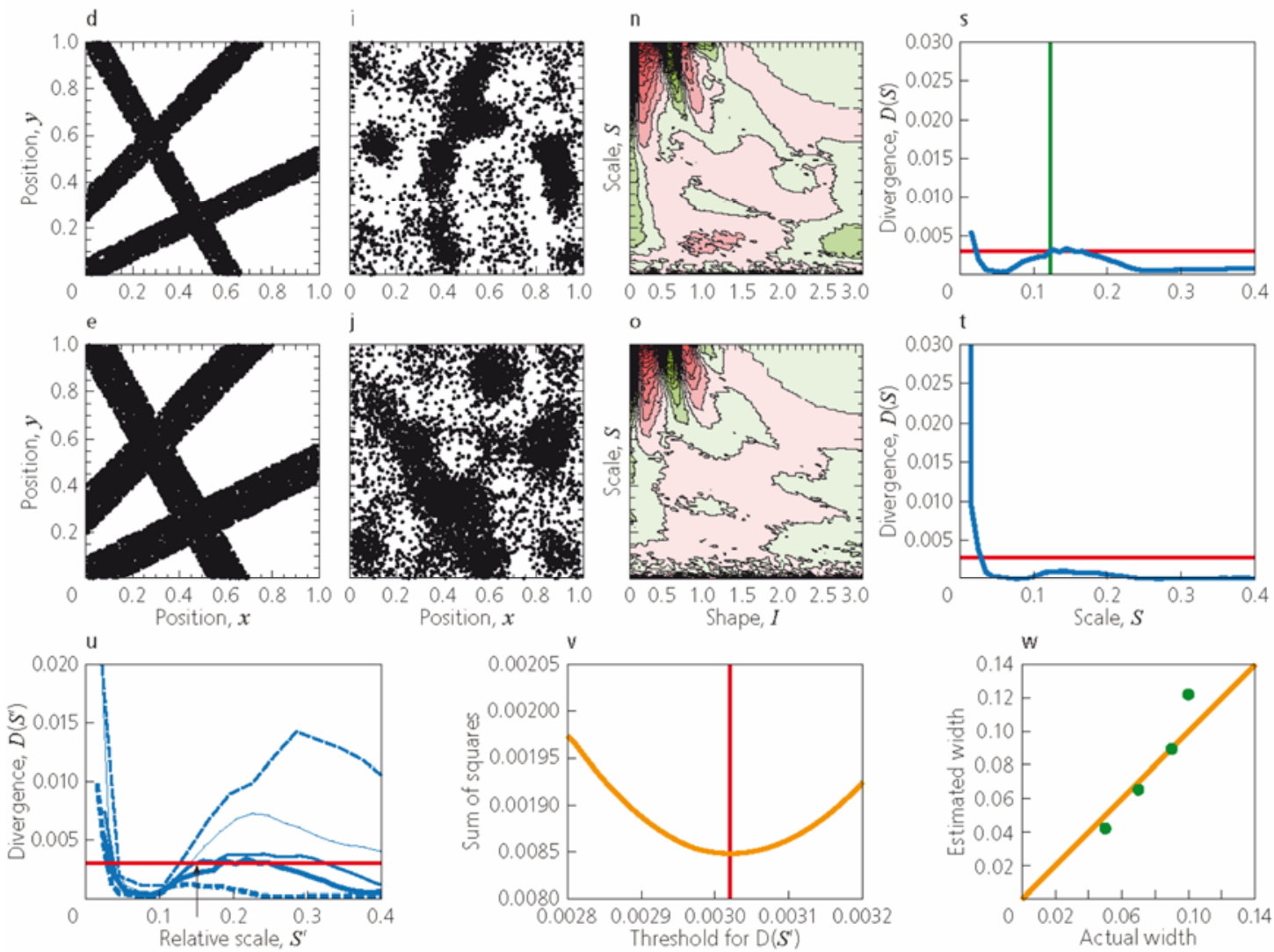


\subsubsection{Point patterns without bandedness}

As a last robustness check, we apply our approach to test patterns that have no apparent bandedness (Figure 9). Figures 9a-c show test patterns with three disks of points, each of which is generated by distributing points under CSR within three disks of radius 0.05 , 0.75 , and 0.1 , respectively. Control patterns, excess triplet-frequency matrices, and the divergence measure $D(S)$ are shown in Figures 9d-f, 9g-i, and 9j-1, respectively. The resultant control patterns, like in the case of ring-shaped bands, are very similar to the original test patterns. For these test patterns, the excess triplet frequencies do not show any tendency of bimodality (Figures $9 \mathrm{~g}-\mathrm{i}$ ), and the divergence measure $D(S)$ is nearly zero at scales comparable to the radii of the disks (Figures 9j-1).

Figure 9.
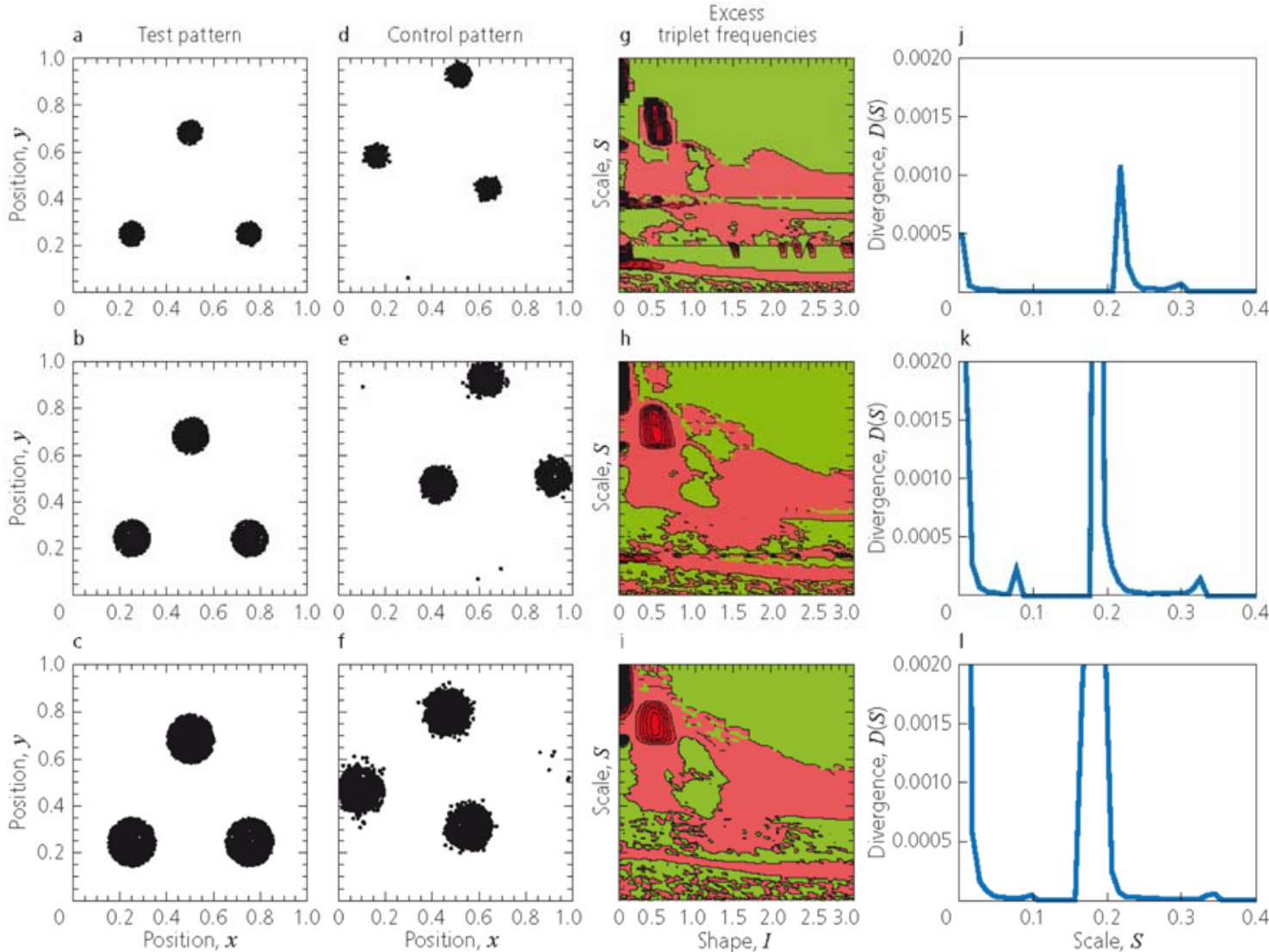

\subsection{Control patterns}

We have stipulated that control patterns against which to compare the frequencies of triplets in a test pattern should have the same first- and second-order structure as the test 
pattern. To generate such control patterns, we use the MH algorithm. A test pattern's thirdorder structure has then been defined by the difference in the triplet frequencies between the test pattern and the control pattern.

It turns out, however, that control patterns, to a greater or a lesser extent, can become similar to the test patterns (Figures 4-9). For test patterns with rectangular bands, arcshaped bands, spiral-shaped bands, and combinations of rectangular bands, some bandedness emerges in the control patterns when the considered bands are narrow. Especially for test patterns with ring-shaped bands and those with three disks of points, both of which are highly symmetric, the generated control patterns are very similar to the test patterns (Figures 6 and 9).

This raises a critical question: Can we reconstruct a point pattern when only using information on its first- and second-order structure? This question is equivalent to the following one: Can we uniquely reconstruct a point pattern, in terms the locations of all its points, from only knowing the number $N$ of points and the $N(N-1) / 2$ inter-point distances between them? It is immediately clear that this can apply, if at all, only up to the rigid translation, rotation, and reflection of the entire point pattern, since inter-point distances are invariant under these transformations and thus contain no information on a pattern's absolute position and orientation. With this proviso, the answer to the last question is trivially yes for a point pattern with $N=3$ points, because the three inter-point distances uniquely determine the original point pattern up to translation, rotation and reflection. For a point pattern with $N=4$ points, it can be shown that the answer is again yes, except for the special case in which one point out of the four is on a line that the other three points determine (see Appendix $\mathrm{C}$ for details). However, the probability of such a special case to be realized in an arbitrary point pattern with $N=4$ points is negligible (it has a probability of "measure zero"). By excluding such a special case, it can thus again be concluded that the first- and second-order structure of a point pattern with $N=4$ points 
uniquely determine it up to rotations, translations, and reflections. Although we are not aware of a proof that extends this argument to point patterns with an arbitrary number of points, studies on multidimensional scaling (Zhang et al. 1994, Cox and Cox 2001) and our control patterns generated by MH (Figures 4-9) suggest that a point pattern's firstand second-order structure may determine it uniquely. If this were true, any control pattern generated for a test pattern would become identical to the test pattern up to rotations, translations, and reflections. In turn, this would render useless our approach to define third-order structure by the difference in triplet frequencies between test and control patterns that agree in their first- and second-order structures. Instead, any higherorder structures would simply be implied by these lower order structures.

It seems clear that reconstructions of the discussed kind are only possible if the information available on a point pattern's second-order structure is perfect, by being both complete and infinitely precise. Thus, the resolution and precision at which second-order information is provided and the accuracy with which control patterns are generated seem to be critical for the reconstruction's feasibility. In practical terms, inter-point distances can never be recorded at infinite resolution or precision and the $\mathrm{MH}$ algorithm cannot be expected to yield perfectly accurate matches between a test pattern's and a control pattern's distributions of inter-point distances.

Therefore, we have examined how the generated control patterns are affected by changing the bin width used to sample and fit a test pattern's and a control pattern's distributions of inter-point distances. In one extreme, this bin width is so large that the generated control pattern simply exhibits CSR with the same first-order structure as the test pattern. In the opposite extreme, with perfect accuracy enabled by an infinitesimal bin with, the control pattern may become identical to the test pattern, although for any larger number of points this is practically impossible to achieve by running the $\mathrm{MH}$ algorithm for a reasonable computation time. In between these two extremes, we generate a series of 
control patterns using various bin widths for a given test pattern to see how the associated accuracy affects the triplet frequencies of control patterns. As accuracy is increased, the generated control patterns are expected to become more similar to the test pattern, so their triplet-frequency matrices are expected to become more and more similar to each other, $\mathbf{T}^{*} \approx \mathbf{T}^{\mathrm{c} *}$

To measure the total divergence $D\left(\mathbf{T}^{*}, \mathbf{T}^{\mathrm{c}^{*}}\right)$ between the two triplet configuration matrices, we again adopt the Jensen-Shannon divergence, $D\left(\mathbf{T}^{*}, \mathbf{T}^{\mathrm{c}^{*}}\right)=D_{\mathrm{JS}}\left(\mathbf{T}^{*} / S_{\max } \| \mathbf{T}^{\mathrm{c} *} / S_{\max }\right)$, where $S_{\max }$ is the number of bins for the scale $s$ (note that after division by $S_{\max }$ the matrix elements of $\mathbf{T}^{*} / S_{\max }$ and $\mathbf{T}^{\mathrm{c}^{*}} / \mathrm{S}_{\max }$ both sum to 1 , because each row of $\mathbf{T}^{*}$ and $\mathbf{T}^{\mathrm{c}^{*}}$ is normalized so as to sum to 1 and there are Smax rows).

Figure 10 shows results for two test patterns, one with a rectangular band (first column of Figure 10a) and the other with a ring-shaped band (first column of Figure 10c). Control patterns generated using bin widths of $0.0015,0.01,0.1$, and 1.5 are shown in the further columns of Figures 10a and 10c, respectively. The corresponding triplet-frequency matrices are shown in Figures $10 \mathrm{~b}$ and 10d, respectively. The corresponding distributions of inter-point distances for the test pattern and the generated control patterns are shown in Figures 10e and 10f, respectively. Figure 10g shows the total divergence in the secondorder structure (defined as the JS divergence of the probability distributions of inter-point distances) as a function of bin width for both test patterns. Figure 10h shows the total divergence $D\left(\mathbf{T}^{*}, \mathbf{T}^{\mathrm{c}^{*}}\right)$ in the third-order structure as a function of bin width for both test patterns.

Figure $10 \mathrm{~g}$ indicates that when using bin widths smaller than 0.03 the second-order structures of the generated control patterns are virtually identical to that of the test pattern. For the test pattern with the rectangular band, a band-like pattern, although not as clear as in the test pattern, emerges as the bin width is narrowed, and for the test pattern with 
the ring-shaped band, a clear ring emerges even for a bin width as large as 0.10 . This again illustrates that a test pattern's first- and the second-order structures can potentially suffice for reconstructing the test pattern. The accuracy required for this, in terms of the bin width used for the matching of second-order structures, may differ depending on the considered test pattern.

Figure $10 \mathrm{~h}$ indicates the existence of a plateau in the dependence on the bin width of the total divergence $D\left(\mathbf{T}^{*}, \mathbf{T}^{\mathrm{c}^{*}}\right)$ in the triplet-frequency matrices. This suggests that defining third-order structure as we have suggested is entirely feasible, provided it is done with an appropriate bin width, a decrease of which no further affects the generated control patterns. For the two test patterns in Figure 10, this holds for bin widths smaller than about 0.03 . Below this threshold, control patterns have nearly identical second-order structure as the test pattern, but their triplet frequencies still stably differ from those of the test pattern.

We can thus conclude that, even though it is theoretically possible that control patterns converge on test patterns (up to translation, rotation, and reflection) as the bin width is decreased (as schematically indicated by the dashed lines in Figure 10h), the extremely small bin widths and the extremely long computation times required for this are practically infeasible. In practice, the bin width can thus safely be set as small as is computationally feasible, without incurring a risk that this would affect the estimated third-order structure. Conversely, using a smaller bin width than that at the right-hand border of the plateau in third-order divergence (as illustrated in Figure 10h, where this border occurs at about 0.03 ) is computationally wasteful, as this bin width is already small enough to ensure the stable estimation of the triplet-frequency matrices, at reduced computational costs. To err on the side of caution, we have therefore opted to use throughout this study a bin width of 0.01 for generating control patterns. 
Figure 10.
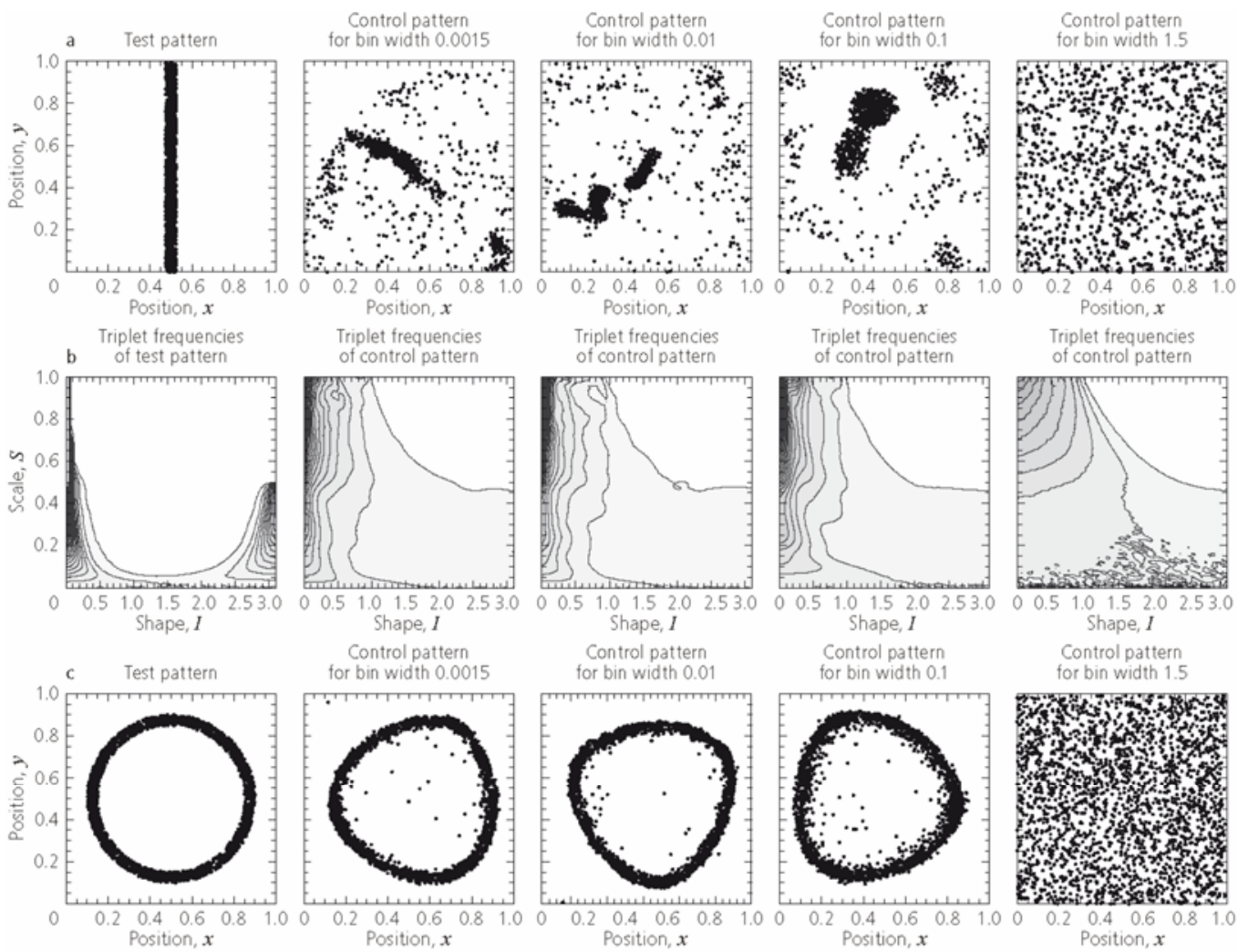

Control pattern

Control pattern
for bin width 0.0
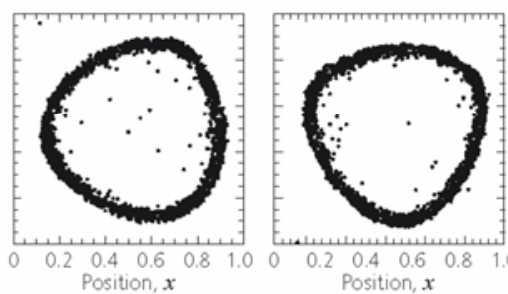

Control pattern
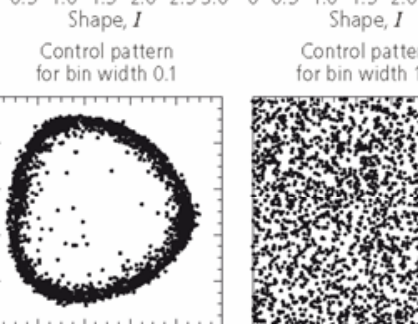

Control pattern
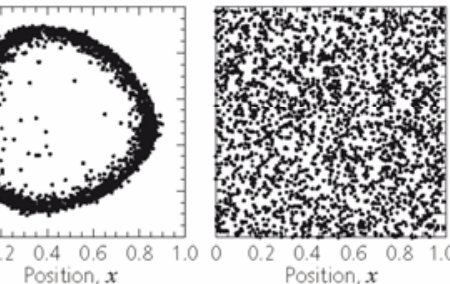

riplet freguencies

of control pattern

Triplet frequencies

Triplet frequencies
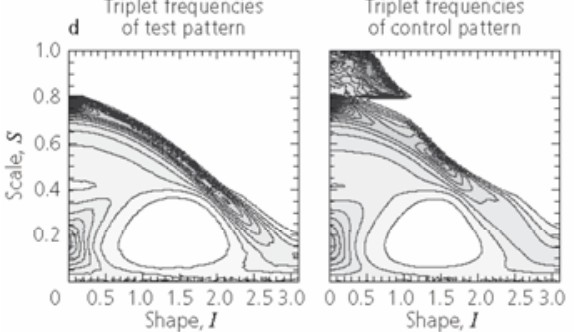

Iriplet frequencies
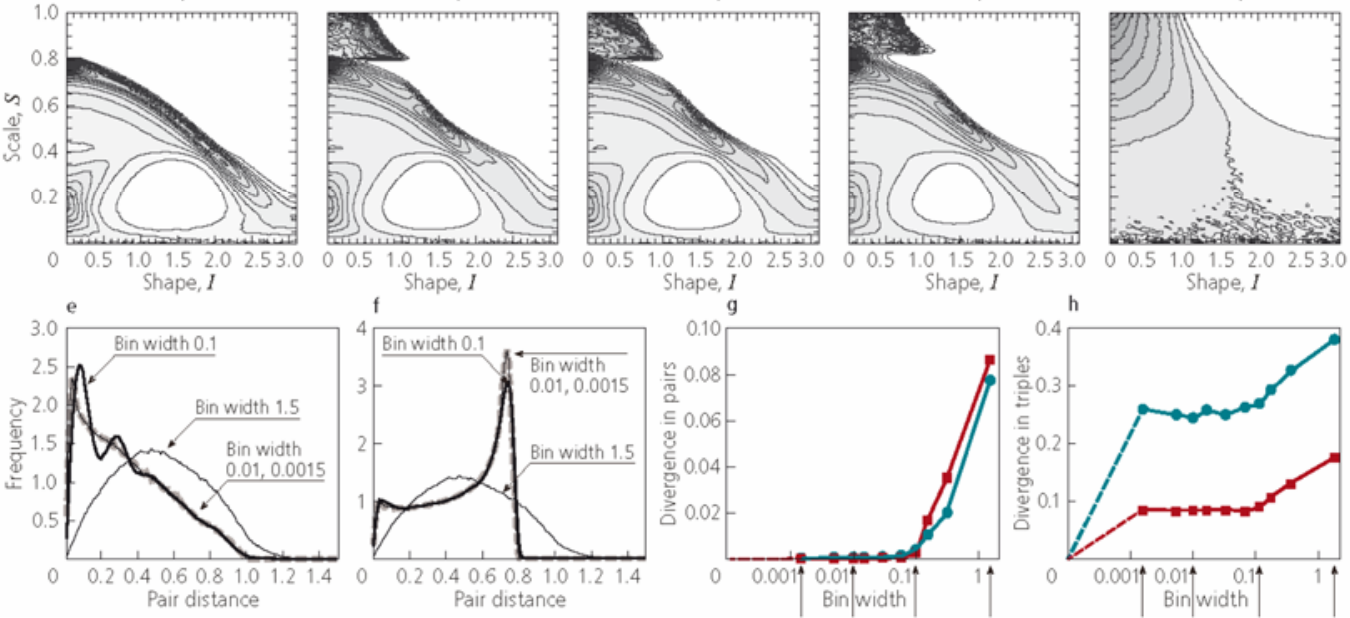

\section{Discussion}

In spatial ecology, increasing attention has been devoted to the characterization of point patterns, leading to the development and application of a variety of tools such as the pair correlation function, Ripley's K function, and O-ring statistics (Ripley 1988, Diggle 2003, Wiegand and Moloney 2004). Focusing only on the number of points, or the singlet density, in the point pattern corresponds to the mean-field approximation that has a long 
history of modeling in the study of population dynamics. The singlet density alone, however, often fails to capture important spatial structures of point patterns we observe in nature, in which interactions between individuals are spatially localized (birth, death, dispersal, intra- and inter-specific competition, etc.) and hence spatial distribution of individuals rarely exhibit CSR even in homogeneous environment. We then need to step into the second order structure defined by pairs. The pair density that carries information of how closely two points are located describes the second order structure and it provides us with a rich array of information about local interactions between individuals as many of previous empirical/theoretical studies have demonstrated.

Intuitively, we may expect that the second-order structure defined by pairs would not be sufficient to describe a point pattern completely. With this expectation, we have defined the third order structure by focusing on triplets as the difference in the triplet configuration when contrasted with that of a control pattern that has the same first and second order structure. Predominance of thinner triplets is expected to highlight bandedness as a typical third order structure in the point pattern (Figure 1). As expected, we have shown that the scale measure $S$ and the shape measure $I$, the arithmetic mean of two edges and the angle surrounded by these, successfully highlight the triplet configurations of point patterns with bandedness and we have developed an approach to derive the characteristic triplet scale hidden in the patterns. Our analysis is heuristic without any probability distributions of the scale and the shape being explicitly derived. However, we stress that our approach to focus on triplets provides a new step for further studies on spatial pattern analyses beyond pairs.

The $\mathrm{MH}$ algorithm we use to generate control patterns has no inherent bias in itself to produce any patterns with orders higher than two and hence we expected that such control patterns will serve as an appropriate reference to gauge the predominance of thinner triplets. It turns out, however, that control patterns become more similar to the test pattern 
as we increase the accuracy to fit the pair density in $\mathrm{MH}$ at least in some highly symmetric cases (Figures 6, 9, and 10). This finding suggests that the first and second order structures may uniquely determine a point pattern and that higher order structures are completely dependent on these lower order structures. If this is true, our approach based on triplet is nullified.

We have shown, however, that there exists a range of accuracy for which generated control patterns are insensitive for changing the accuracy (Figure 10) and that bandedness as a typical third order structure can be still highlighted using the difference in the triplet configuration; with this accuracy, the distribution of shapes starts to diverge above a characteristic triplet scale that is linearly related to the band width of point patterns (Figures 4-8). For some test pattern like a ring, control patterns become very similar to the test pattern (Figure 6). Similarity in the test and the control pattern consequently results in a very small optimal divergence threshold of 0.0001645 for patterns with a ring, compared with other patterns with a band, an arc, a spiral and crosses, threshold for each of which is $0.0043,0.0046,0.0122$, and 0.00302 , respectively. Nonetheless, our approach based on triplets successfully highlights banded structure by focusing on the predominance of thinner triplet at a certain scale.

We here summarize our conjecture on the dependency of control patterns on the accuracy to fit the pair density and on the resultant extractions of the third order structure in Table 1. There remain lots of things to be learned about the use of control patterns to gauge thinner triplets and to define the third order structure.

Table 1. Accuracy in sampling and matching the second-order structures (pair densities) of a test pattern and a control pattern and its consequence for identifying the test pattern's third-order structure (triplet density).

\begin{tabular}{|l|l|}
\hline Perfect accuracy & $\begin{array}{l}\text { The control pattern has exactly the same first- and second- } \\
\text { order structure as the test pattern, and is thus identical to it (up }\end{array}$ \\
\hline
\end{tabular}




\begin{tabular}{|c|c|}
\hline & $\begin{array}{l}\text { to translations, rotations, and reflections). Accordingly, there } \\
\text { is no difference in the triplet densities of the test pattern and } \\
\text { the control pattern. If a test pattern's third-order structure were } \\
\text { defined based on such control patterns, it would be completely } \\
\text { determined by its first- and second-order structures, and could } \\
\text { thus contain no independent extra information. }\end{array}$ \\
\hline Intermediate accuracy & $\begin{array}{l}\text { The control pattern has exactly the same first-order structure } \\
\text { and very nearly the same second-order structure as the test } \\
\text { pattern. The control pattern is safely prevented from becoming } \\
\text { identical to the test pattern (up to translations, rotations, and } \\
\text { reflections) by using bins of finite width in sampling and } \\
\text { matching the control pattern's pair density to that of the test } \\
\text { pattern. (Alternatively, the control pattern's pair density may } \\
\text { be sampled without including all pairs, or the matching } \\
\text { process may be stopped shortly before perfect convergence is } \\
\text { reached.) The test pattern's third-order structure, defined by } \\
\text { the difference in the triplet densities of a test pattern and such } \\
\text { control patterns, thus contains independent extra information, } \\
\text { which can be used, e.g., for detecting bandedness in the test } \\
\text { pattern. }\end{array}$ \\
\hline No accuracy & $\begin{array}{l}\text { The control pattern has exactly the same first-order structure } \\
\text { as the test pattern, but no second-order structure, as its points } \\
\text { are distributed according to CSR. If a test pattern's third-order } \\
\text { structure were defined based on such control patterns, it would } \\
\text { contain a contribution from the difference in the test pattern's } \\
\text { and control pattern's pair densities. This would mean that the } \\
\text { test pattern's third-order structure could not be defined } \\
\text { independently from its second-order structure. }\end{array}$ \\
\hline
\end{tabular}


When we derived optimal divergence thresholds, we rescaled the divergence in the shape distribution $D\left(S^{\prime}\right)$ (Figures $4 \mathrm{u}-8 \mathrm{u}$ ) and calculated the sum of squared deviations from a reference band width of 0.15 , an arbitral value. But this rescaling should work perfectly only for a vertically infinitely long straight band. Furthermore, for a test pattern with a curved band, curvature of the band relative to the band width would critically affect the optimal divergence threshold because triangles that have the same shape measure but are larger in scale are possible for such a curved band (cf. optimal divergence threshold for an arc 0.0046 is larger than that for the straight band 0.0043). For this reason, our derivation of optimal divergence thresholds based on a fixed reference width of 0.15 should be considered as a crude method that has much room to be improved. Inconsistency in the magnitude of the optimal divergence thresholds for the five test patterns we tested is likely related to this issue and also to how generated control patterns become similar to the original test pattern, which will be linked with the degree of symmetry of the test pattern and the algorithm we use to fit the second order structure. Further study is needed to explain the large variation in the optimal divergence thresholds.

Schladitz and Baddeley (2000) examined a third order structure by focusing on the $r$ close triplets and derived the expected number of $r$-close triplets for the homogeneous Poisson process and the Gauss-Poisson process. But their motivation was not to detect bandedness in point pattern. They also did not explicitly considered the triplet configurations to explore triplet "shape". Dale and Powell (2001) developed a method that focuses on the points within the circumcircle made by a triplet. Their focus was to detect gaps in a point pattern and they also did not explore "shape" of a triplet, though the radius of the circumscribed circle in which there are fewer points corresponds to gap size. Our approach is similar to Dale and Powell (2001) but differs essentially in that we explicitly consider the spatial configuration of a triplet by its scale and shape and reduces the dimensionality of triplet configuration. 
Dimensionality, or degree of freedom, of a triplet is three in isotropic pattern. Therefore, a triplet cannot be uniquely determined by a scale and a shape measure. In order to simplify the analysis, however, reduction of the dimensionality is necessary. Furthermore, any attempt to simplify spatial complexity is worth to carry out and should be pursued (Dieckmann et al. 2000). In this paper we focus on triplets and measure a triplet in terms of the scale and shape as a two dimensional measure.

The scale and shape of a triplet can be defined in various ways. For examples, the maximum of two edges, the radius of the inscribed circle of the triplet, the radius of the circumscribed circle, the arithmetic mean of the three edges, the maximum of the three edges, can be a measure of scale. In the same way, other measures of shape are possible; the ratio of the inscribed circle radius to the sum of three edges and the ratio of the minimum of triangle height to the maximum edge can be a measure of shape. Further study is needed to explore which combination of scale and shape measures can be useful to better highlight bandedness as a typical third order structure.

Our approach based on triplets focuses on the predominance of thinner triplets in a point pattern. As we have shown for the five types of test patterns, our approach should be able to highlight bandedness with some directionality as long as the band width is constant and narrow enough. If the band width is large relative to the distance with another band, or if the band width varies along a band, there are less thinner triangles in the point pattern and our approach will fail as well as for point patterns that have no directionality like disks. Further study is needed to simplify the complexity of point pattern in terms of triplets.

We have a priori assumed that the next structure beyond pairs is represented by triplets. Triplets, however, might not necessarily be the next level structural component. A 
possible way is to consider a conditional probability that a point in a pair makes another pair with another point to form an open triangle. This extended pair-based approach may work to highlight banded patterns. It, however, remains an open question how to quantity such an open triangle and to deal with the conditionality. If our conjecture that the second order structure uniquely determines the point pattern holds true, this extended pair-based approach may successfully capture the spatial configuration of point patterns. Further study is needed to quantify and simplify triplet configurations in terms of the lower order structure.

So far, a very few attempts have been made that focus on triplets in point pattern analysis primarily because of the difficulty with which we measure the scale and the shape of a triplet and interpret the triplet configuration in terms of the predominance of thinner triplets. We have responded to this challenge and have shown that a simple scale and shape measure can highlight bandedness as a typical third order structure of a point pattern. Our approach could be applied to a variety of point patterns observed in the real world such as bandedness generated by wave-regeneration in forest ecology (Sprugel 1976, Iwasa et al. 1991, Sato and Iwasa 1993, Shibuya et al. 2004). There seem to be few studies that focus on bandedness with some directionality in point pattern analysis. In order to better understand the characteristics of point patterns and to pursue further simplification of spatial complexity, we call for further study that focuses on triplets beyond pairs.

\section{Acknowledgements}

We thank Dr. Kenichiro Shimatani for valuable comments on earlier versions of our analysis of triplet configurations. We also thank two anonymous reviewers for their valuable comments and suggestions to improve this paper. This study was supported by JSPS Grant-in-Aid for Scientific Research (C) 23570026 to FT. This study was further supported by grant TECT I-106 G11 from the Austrian Science Fund to UD for the 
research project The Adaptive Evolution of Mutualistic Interactions as part of the multinational collaborative research project Mutualisms, Contracts, Space, and Dispersal (BIOCONTRACT) selected by the European Science Foundation as part of the European Collaborative Research (EUROCORES) Programme The Evolution of Cooperation and Trading (TECT). UD gratefully acknowledges additional support by the European Commission, the European Science Foundation, the Austrian Ministry of Science and Research, and the Vienna Science and Technology Fund. 


\section{Appendix A: Generating control patterns using the Metropolis-Hastings algorithm}

2 We use the Metropolis-Hastings $(\mathrm{MH})$ algorithm to generate a control pattern that has the

3 same first- and second-order structure as a test pattern.

4

5 We start by determining the pair density (distribution of inter-point distances) of the test pattern, using the specified bin width $b$. All pairs are sampled and the frequency $p_{i}$ of

7 pairs falling within the $i$ th bin is obtained, normalized so that $\sum_{i} b p_{i}=1$.

9 We then initialize the $\mathrm{MH}$ algorithm with a CSR point pattern that covers the same range $10(0 \leq x \leq 1,0 \leq y \leq 1)$ as the test pattern and that has the same number of points as the test 11 pattern. To refine this control pattern successively using the $\mathrm{MH}$ algorithm, we repeat the 12 following steps until the pair densities of the control pattern has sufficiently converged to 13 that of the test pattern:

15 (1) Calculate the pair density of the current control pattern using the bin width $b$. All pairs 16 are sampled and the frequency $p_{c i}$ of pairs falling within the $i$ th bin is obtained, normalized 17 so that $\sum_{i} b p_{\mathrm{ci}}=1$

(2) Randomly choose a point of the control pattern and randomly shift its location $(x, y)$ to $\left(x^{\prime}, y^{\prime}\right)$ to create a trial control pattern, where $x^{\prime}$ and $y^{\prime}$ are independent random numbers uniformly distributed between 0 and $1, x^{\prime}, y^{\prime} \sim U[0,1)$. Calculate the pair density of the trial control pattern using the bin width $b$. All pairs are sampled and the frequency $p_{\mathrm{c} i}^{\prime}$

23 of pairs falling within the ith bin is obtained, normalized so that $\sum_{i} b p_{\mathrm{c} i}^{\prime}=1$.

(3) Calculate the sums $D$ and $D^{\prime}$ of squared differences between the pair density of the test pattern and the pair densities of the current control pattern and trial control pattern, 
respectively,

$$
D=\sum_{i} b\left(p_{i}-p_{\mathrm{ci}}\right)^{2} \text { and } D^{\prime}=\sum_{i} b\left(p_{i}-p_{\mathrm{ci}}^{\prime}\right)^{2}
$$

Calculate the probability of accepting the trial control pattern as

$$
P=\left[1+e^{s\left(D^{\prime}-D\right) / D}\right]^{-1},
$$

31 where the parameter $s>0$ controls the convergence speed (we use $s=1.0 \times 10^{4}$ ). With probability $P$, accept the trial control pattern as the new current control pattern. Otherwise, reject the trial and leave the current control pattern unchanged.

(4) Repeat (1) through (3) until $p$ converges to $p_{c}$ to a specified degree, so that the sum $D$ of squared differences becomes smaller than a threshold $D_{\min }$ (we use $D_{\min }=1.0 \mathrm{x}$ $\left.10^{-4}\right)$.

Control patterns generated in this way have exactly the same first-order structure and very nearly the same second-order structure as the test pattern, with the bin width $b$ and the convergence threshold $D_{\min }$ controlling the accuracy of the latter match. When $D_{\min }$ is chosen sufficiently small, the match's accuracy is thus controlled by $b$.

For finite bin width $b$, control patterns independently generated in this way differ from each other (as well as from the test pattern). When the bin width $b$ is chosen sufficiently small, the triplet-frequency matrix $\mathbf{T}^{\mathrm{c}^{*}}$ becomes essentially independent of $b$. To improve accuracy, we generate 10 control patterns for each test pattern; the triplet-frequency matrix $\mathbf{T}^{\mathbf{c}^{*}}$ is then calculated as an average over these 10 control patterns.

\section{Appendix B: Determining Jensen-Shannon divergence}

The Jensen-Shannon divergence $\operatorname{DJs}(P \| Q)$ measures the divergence between two probability distributions $P=\left(P_{i}\right)$ and $Q=\left(Q_{i}\right)$ and is defined as 


$$
D_{\mathrm{JS}}(P \| Q)=\frac{1}{2} D_{\mathrm{KL}}(P \| M)+\frac{1}{2} D_{\mathrm{KL}}(Q \| M),
$$

where $M=(P+Q) / 2$ and $D_{K L}(P \| Q)$ is the Kullback-Leibler $(\mathrm{KL})$ divergence defined as

$$
D_{\mathrm{KL}}(P \| Q)=\sum_{i} P_{i} \ln \left(P_{i} / Q_{i}\right)
$$

60

To measure the divergence $D(S)$ in the frequency distributions of triplet shapes $I$ as a

62 function of triplet scale $S$, we set $P_{i}=\mathbf{T}^{*}{ }_{s i}$ and $Q_{i}=\mathbf{T}^{\mathrm{c}^{*}}{ }_{s i}$ (where $s$ and $i$ are the bins corresponding to $S$ and $I$ ). We use the JS divergence because (in contrast to the KL) divergence) it is symmetric, so it can be considered as a metric distance between two distributions.

66

Appendix C: Reconstructing patterns of four points using perfect second-order information

69 We consider a triangle OAB in two-dimensional space. Without loss of generality, we set $\mathrm{O}=(0,0), \mathrm{A}=\left(a_{1}, a_{2}\right)$, and $\mathrm{B}=\left(b_{1}, b_{2}\right)$. We can add a new point $\mathrm{C}=\left(c_{1}, c_{2}\right)$ that creates a parallelogram $\mathrm{ACA}^{\prime} \mathrm{B}$ with another point $\mathrm{A}^{\prime}$ that satisfies $\mathrm{OA}=\mathrm{OA}^{\prime}$, i.e., $\mathrm{A}^{\prime}$ is located on the circle centered on $\mathrm{O}$ with radius $\mathrm{OA}$. Then the point pattern $\mathrm{OABC}$ and $\mathrm{OA}^{\prime} \mathrm{BC}$, although differing from each other, have the same inter-point distances. For this to occur, however, the point $C$ has to satisfy $\left(b_{1}+c_{1}\right)^{2}+\left(b_{2}+c_{2}\right)^{2}=2 a_{1}\left(b_{1}+c_{1}\right)+2 a_{2}\left(b_{2}+c_{2}\right)$, i.e., on a circle whose measure is zero in two dimensional space. Therefore, the probability that a point pattern comprising four arbitrary points has another point pattern with identical inter-point distances can be considered zero, i.e., a point pattern with $N=4$ can be uniquely constructed using only inter-point distances. 


\section{Literature cited}

B. Bhanu, X. Tan, 2001, A Triplet Based Approach for Indexing of Fingerprint Database for Identification, In J. Bigun and F. Smeraldi (Eds.): Audio- and Video-Based Biometric Person Authentication, Third International Conference, AVBPA 2001 Halmstad, Sweden, June 6-8, 2001 Proceedings, Lecture Notes in Computer Science 2091:205-210.

B. M. Bolker, S. W. Pacala. 1997. Using moment equations to understand stochastically driven spatial pattern formation in ecological systems. Theoretical Population Biology 52:179-197.

B. M. Bolker, S. W. Pacala, 1999, Spatial Moment Equations for Plant Competition: Understanding Spatial Strategies and the Advantages of Short Dispersal, American Naturalist 153:575-602.

R. Condit, 1998, Tropical Forest Census Plots: Methods and Results from Barro Colorado Island, Panama and a Comparison with Other Plots, Springer.

T. F. Cox, M. A. A. Cox, 2001, Multidimensional Scaling, CRC Press.

N. A. C. Cressie, 1993, Statistics for spatial data, Wiley series in probability and mathematical statistics, John Wiley \& Sons, Inc.

M. R. T. Dale, 1999, Spatial pattern analysis in plant ecology. Cambridge University Press, Cambridge.

M. R. T. Dale, R. D. Powell, 2001, A new method for characterizing point patterns in plant ecology, Journal of Vegetation Science 12:597-608.

U. Dieckmann, R. Law, J. A. J. Metz eds., 2000, The geometry of ecological interactions: simplifying spatial complexity, Cambridge studies in adaptive dynamics, Cambridge University Press.

P. J. Diggle, 2003, Statistical Analysis of Spatial Point Patterns, 2nd edn. Oxford: Oxford University Press.

P. Grieg-Smith, 1983, Quantitative plant ecology. Studies in Ecology Vol. 9. University 
of California Press.

108 W. K. Hastings, 1970, Monte Carlo Sampling Methods Using Markov Chains and Their Applications, Biometrika 57:97-109.

J. Illian, A. Penttinen, H. Stoyan, D. Stoyan. 2008. Statistical Analysis and Modelling of Spatial Point Patterns. Chichester, England, John Wiley \& Sons.

112 Y. Iwasa, K. Sato, S. Nakashima, 1991, Dynamic modeling of wave regeneration (Shimagare) in subalpine Abies forests, Journal of Theoretical Biology 152:143158.

J. T. Kerr, M. Ostrovsky, 2003, From space to species: ecological applications for remote sensing, Trends in Ecology and Evolution 18:299-305.

R. Law, U. Dieckmann, 2000, A dynamical system for neighborhoods in plant communities, Ecology 81:2137-2148.

R. Law, D. J. Murrell, U. Dieckmann, 2003, Population growth in space and time: spatial logistic equations, Ecology 84:252-262.

R. Law, J. Illian, D. F. R. O. Burslem, G. Gratzer, C. V. S. Gunatilleke, I. A. U. N. Guantilleke, 2009, Ecological information from spatial patterns of plants: insights from point process theory, Journal of Ecology 97:616-628.

S. A. Levin, 1992, The problem of pattern and scale in ecology: The Robert H. MacArthur Award Lecture, Ecology 73:1943-1967.

A. M. Liebhold, J. Gurevitch, 2002, Integrating the statistical analysis of spatial data in ecology, Ecography 25:553-557.

128 V. V. Orlov, A. V. Petrova, V. G. Tarantaev, 2001, The intrinsic shapes of galaxy groups, Mon. Not. R. Astron. Soc. 325:133-137.

130 E. C. Pielou, 1969, An introduction to mathematical ecology, Wiley, New York.

131 B. D. Ripley, 1988, Statistical Inference for Spatial Processes. Cambridge University Press, Cambridge.

J. Roughgarden, S. W. Running, P. A. Matson, 1991, What does remote sensing do for 

ecology?, Ecology 72:1918-1922.

135 K. Sato, Y. Iwasa, 1993, Modeling of wave regeneration in subalpine Abies forests: 136 population dynamics with spatial structure, Ecology 74:1538-1550.

137 K. Schladitz, A. J. Baddeley, 2000, A third order point process characteristic, 138 Scandinavian Journal of Statistics 27:657-671.

139 M. Shibuya, N. Haga, T. Sasaki, S. Kikuchi, M. Haruki, M. Noda, K. Takahashi, K. 140 Matsuda, 2004, Stand and self-thinning dynamics in natural Abies stands in northern Hokkaido, Japan, Ecological Research 19:301-309.

142 K. Shimatani, Y. Kubota, 2004, Spatial analysis for continuously changing point patterns 143 along a gradient and its application to an Abies sachalinensis population, $144 \quad$ Ecological Modelling 180:359-369.

145 D. G. Sprugel, 1976, Dynamic Structure of Wave-Regenerated Abies balsamea Forests in the North- Eastern United States. Journal of Ecology 64:889-911.

147 D. Tilman, P. Kareiva, 1997, Spatial ecology: the role of space in population dynamics 148 and interspecific interactions. Princeton, NJ, USA, Princeton University Press.

149 M. G. Turner, 1989, Landscape ecology: the effect of pattern on process, Annual Review of Ecology and Systematics 20:171-197.

151 A. S. Watt, 1947, Pattern and process in the plant community. Journal of Ecology 35:122.

153 T. Wiegand, K. A. Moloney, 2004, Rings, circles, and null-models for point pattern analysis in ecology, Oikos 104:209-229.

155 T. Wiegand, T. Gunatilleke, N. Gunatilleke, 2007a, Species associations in a 156 heterogeneous Sri Lankan Dipterocarp forest. American Naturalist 170:E77-E95. 157 T. Wiegand, T. Gunatilleke, N. Gunatilleke, T. Okuda, 2007b, Analysing the spatial 158 structure of a Sri Lankan tree species with multiple scales of clustering. Ecology 88:3088-3102.

160 Y. Y. Zhang, S. H. Levine, J. G. Kreifeldt, 1994. Point pattern reconstruction with less 
Information and Technology'. Vol. 1. IEEE, 1994.

163

164 
167 Figure 1. Illustration of how triplet densities help identify bandedness in point patterns.

168 If a point pattern contains bands of points, thinner triplets predominate at a characteristic

169 scale determined by the width of the band. The example shows a spatial pattern given by a spiral-shaped band of points. Triplets are formed by any three points randomly chosen from the point pattern. (a) Triplets of all shapes occur at large scales. (b) Thinner triplets predominate at intermediate scales comparable to the width of the band. (c) Triplets of all shapes again occur at smaller scales. This shows how the analysis of third-order structure, by considering triplet shapes across scales, reveals extra information about a point pattern (and in particular, its bandedness) that cannot be inferred from the pattern's second-order structure (which essentially can only reveal aggregation or segregation).

Figure 2. Spatial configuration of a triplet comprising three distinct points $i, j, k$ of a point pattern, with its three edges $L_{i}, L_{j}, L_{k}$ and three angles $\theta_{i}, \theta_{j}, \theta_{k}$.

Figure 3. Deriving third-order structure by comparing the triplet frequencies of a test pattern with those of a control pattern. (a) A test pattern with a rectangular band of points: 3,000 points are distributed under complete spatial randomness (CSR) within a rectangle of width 0.15 and height 1 embedded in an otherwise empty square with edges of length $1(0 \leq x \leq 1,0 \leq y \leq 1)$. (b) A matching control pattern, generated by starting from an initial pattern with CSR and the same first-order structure (number of points) as the test pattern by successively moving individual points using the Metropolis-Hastings (MH)

188 algorithm so as to bring its second-order structure (pair density) sufficiently close to that of the test pattern. (c) Pair densities as a function of pair distance for the test pattern

190 (dashed line) and the control pattern (continuous line). Also shown are the pair densities

191 for the CSR pattern used to initialize the MH algorithm (thin line). (d) The triplet 
192 frequencies of the test pattern, represented as a contour plot of the triplet-frequency matrix

$193 \mathbf{T}^{*}$. The measure $I$ of triplet shape (angle between triplet edges in radians) varies along

194 the horizontal axis, while the measure $S$ of triplet scale (arithmetic mean of the corresponding edges) varies along the vertical axis. (e) The corresponding triplet

196 frequencies of the control pattern, represented as a contour plot of the triplet-frequency

197 matrix $\mathbf{T}^{\mathrm{c}^{*}}$. (f) Contour plot of the excess triplet-frequency matrix $\mathbf{T}^{\mathrm{e}^{*}}=\mathbf{T}^{*}-\mathbf{T}^{\mathrm{c}^{*}}$.

198 Excesses of triplets are shown in green (i.e., for these combinations of $I$ and $S$, the test

199 pattern has more triplets than the control pattern), while shortages of triplets are shown

200 in red. The zero contour separating regions of excess and shortage is shown as a thick

201 line. (g) Jensen-Shannon (JS) divergence $D(S)$ of the triplet-frequency matrices of test

202 pattern and control pattern as a function of the scale $S$.

203

204 Figure 4. Third-order structure of rectangular bands of widths $w=0.05$ (a), 0.10 (b),

$2050.15(\mathrm{c}), 0.20(\mathrm{~d})$, and 0.25 (e). The number of points is $1,000,2,000,3,000,4,000$, and 5,000 points, respectively, so that the singlet density within the bands is kept constant despite the change of band width. (f)-(j) Corresponding control patterns having the same first- and second-order structure as the test patterns. (k)-(o) Corresponding excess triplet frequencies. (p)-(t) Corresponding divergence $D(S)$ of the frequency distributions of triplet shapes as a function of the triplet scale $S$. The optimal divergence threshold and

211 the resultant estimates of the characteristic triplet scales are shown as horizontal and

212 vertical lines, respectively. (u) Divergences $D\left(S^{\prime}\right)$ as functions of the relative scale $S^{\prime}$ that

213 is standardized to the reference width of 0.15 (the functions shown as thin dashed, thin

214 continuous, continuous, thick, and thick dashed lines correspond to the band widths 0.05 ,

$2150.10,0.15,0.20$, and 0.25 , respectively). The optimal divergence threshold is shown as a

216 horizontal line. The reference band width of 0.15 is indicated by the vertical arrow. (v)

217 Sum of squares of the deviations between the estimated relative characteristic triplet 218 scales and the reference band width of 0.15 as functions of a continuum of candidate 

the actual band widths.

Figure 5. Third-order structure of arc-shaped bands of widths $w=0.05$ (a), 0.10 (b), 0.15 (c), 0.20 (d), and 0.25 (e). Points are distributed under complete spatial randomness (CSR) within a ring-shaped band, $r_{\text {in }}^{2}<(x+1 / 2)^{2}+(y-1 / 2)^{2}<r_{\text {out }}^{2} \quad$ with $r_{\text {in }}=1-w / 2$ and $r_{\text {out }}=1+w / 2$, that is clipped to an arc-shaped band within $0 \leq x \leq 1$ and $0 \leq y \leq 1$. The number of points is $1,048,2,062,3,229,4,249$, and 5,244 , respectively, so that the singlet density within the bands is kept constant despite the change of band width. Other details as in Figure 4.

229

Figure 6. Third-order structure of ring-shaped bands of widths $w=0.05$ (a), 0.07 (b), $0.09(\mathrm{c}), 0.10(\mathrm{~d})$, and $0.15(\mathrm{e})$. Points are distributed under complete spatial randomness (CSR) within a ring-shaped band, $r_{\text {in }}^{2}<(x-1 / 2)^{2}+(y-1 / 2)^{2}<r_{\text {out }}^{2}$ with $r_{\text {in }}=0.4-w$ and $r_{\text {out }}=0.4$. The number of points is 2,357, 3,211, 4,015, 4,399, and 6,127, respectively, so that the singlet density within the bands is kept constant despite the change of band width. Other details as in Figure 4.

Figure 7. Third-order structure of spiral-shaped bands of widths $w=0.02(\mathrm{a}), 0.03(\mathrm{~b})$, 0.04 (c), 0.05 (d), and 0.06 (e). Points are distributed under complete spatial randomness (CSR) within a spiral-shaped band generated as follows. Using independent random numbers uniformly distributed between 0 and $1, R_{1 i}, R_{2 i}, R_{3 i}: U[0,1)$, a series of points $\left(x_{i}, y_{i}\right)$ is generated as $x_{i}=\left(\phi_{i} \cos \phi_{i}\right) / 6 \pi+d_{i} \cos \alpha_{i}, \quad y_{i}=\left(\phi_{i} \sin \phi_{i}\right) / 6 \pi+d_{i} \sin \alpha_{i}$, with $\phi_{i}=6 \pi \sqrt{R_{1 i}}, d_{i}=w \sqrt{R_{2 i}}$, and $a_{i}=2 \pi R_{3 i}$. The first terms on the right-hand sides of $x_{i}$ and $y_{i}$ describe an Archimedean spiral, while the second terms describe points distributed under CSR within a band following this spiral. The number of points is 1,906 , $2,860,3,813,4,767$, and 5,720 , respectively, so that the singlet density within the bands 
is kept constant despite the change of band width. Other details as in Figure 4.

248 Figure 8. Third-order structure of crosses of three rectangular bands of widths $w=0.05$ (a), 0.07 (b), 0.09 (c), 0.10 (d), and 0.15 (e). Points are distributed under complete spatial randomness (CSR) within the three rectangular bands generated as follows. Using independent random numbers uniformly distributed between 0 and $1, x_{i}, y_{i}: U[0,1)$, a series of points $\left(x_{i}, y_{i}\right)$ is generated by accepting points for which the distance between the point and at least one of three lines $\left(x-x_{j}^{*}\right) \sin a_{j}-\left(y-y_{j}^{*}\right) \cos a_{j}=0$ is less than $w / 2$. The three lines are specified by $a_{1}=p / 4,\left(x_{1}^{*}, y_{1}^{*}\right)=(0.5,0.8), a_{2}=-p / 3$, $\left(x_{2}^{*}, y_{2}^{*}\right)=(0.5,0.2)$, and $a_{3}=p / 7,\left(x_{3}^{*}, y_{3}^{*}\right)=(0.8,0.4)$. The number of accepted points is set to $3,000,4,200,5,400,6,000$, and 9,000 , respectively, so that the singlet density within the bands is kept nearly constant despite the change of band width. Other details as in Figure 4.

Figure 9. Third-order structure of three disks with radii $w=0.05(\mathrm{a}), 0.075(\mathrm{~b})$, and 0.1 (c). Points are distributed under complete spatial randomness (CSR) within disks centered at $(0.25,0.25),(0.75,0.25)$, and $(0.5,0.683)$. The number of points is set to $630,1,416$, and 2,514, respectively, so that the singlet density within the disks is kept constant despite the change of disk radius. Other details as in Figure 4.

Figure 10. Dependence of the triplet frequencies of a control pattern on the accuracy with which the pair densities of a test pattern and a control pattern are sampled and matched. (a) \& (c) Two test patterns given by, respectively, a rectangular band of width 0.05 and by a ring-shaped band of width 0.05 (left column) and corresponding control patterns (other columns) obtained for different bin widths for sampling and matching the pair densities. From left to right, the bin width decreases from 1.5, to 0.1, 0.01, and 0.0015. (b) \& (d) Corresponding triplet-frequency matrices of the test pattern and control patterns. 
274 pattern and control patterns (rectangular band and ring-shaped band, respectively). Thick 275 dashed gray curves show the pair density of the test pattern and thick continuous black 276 curves show those for the control patterns (the corresponding bin widths are distinguished 277 by labeled arrows). (g) Divergence in second-order structure as a function of bin width 278 (the sampled bin widths are $0.0015,0.005,0.01,0.015,0.03,0.05,0.10,0.15,0.3$, and 279 1.5). Filled circles correspond to the rectangular band and filled squares to the ring-shaped 280 band. The arrows indicate the bin widths of $0.0015,0.01,0.1$, and 1.5 shown in (a) to (f). 281 Note that the horizontal axis is logarithmically scaled. (h) Total divergence in third-order 282 structure as a function of bin width. Other details as in $(\mathrm{g})$. Our conjecture that perfect 283 accuracy (corresponding to an infinitesimal bin width) would typically result in control 284 patterns that are identical to test patterns (up to translation, rotation, and reflection), so 285 that the total divergence becomes zero, is schematically indicated by the dashed lines. 